\title{
Transumanza e territorio in Toscana: percorsi e pascoli \\ dalla Protostoria all'Età contemporanea
}

La strutturazione del sistema informativo e le attività di ricognizione archeologica (2016)

Giovanna Pizziolo, Michele De Silva, Nicoletta Volante e Davide Cristoferi

\section{(2) OpenEdition}

\section{Journals}

Edizione digitale

URL: http://journals.openedition.org/cefr/1836

DOI: $10.4000 /$ cefr. 1836

ISSN: 2282-5703

Editore

École française de Rome

Notizia bibliografica digitale

Giovanna Pizziolo, Michele De Silva, Nicoletta Volante e Davide Cristoferi, « Transumanza e territorio in Toscana: percorsi e pascoli

dalla Protostoria all'Età contemporanea », Chronique des activités archéologiques de l'École française de Rome [Online], Italie centrale, Messo online il 31 octobre 2017, consultato il 06 août 2019. URL : http:// journals.openedition.org/cefr/1836; DOI : 10.4000/cefr.1836

Questo documento è stato generato automaticamente il 6 agosto 2019

(c) École française de Rome 


\section{Transumanza e territorio in Toscana: percorsi e pascoli dalla Protostoria all'Età contemporanea}

La strutturazione del sistema informativo e le attività di ricognizione archeologica (2016)

Giovanna Pizziolo, Michele De Silva, Nicoletta Volante e Davide Cristoferi

\section{NOTE DELL'AUTORE}

Il gruppo di lavoro del progetto TraTTo (TRAsumanza e Territorio in Toscana) è costituita da membri e collaboratori del Dipartimento di Scienze Storiche e del Beni Culturali dell'Università di Siena: Prof. Andrea Zagli (storico, responsabile scientifico), Franco Cambi (archeologo), Carlo Citter (archeologo), Davide Cristoferi (storico, referente Medioevo-Età Moderna), Michele De Silva (geografo, referente GIS), Anna Guarducci (geografa), Giancarlo Macchi Jánica (geografo), Giovanna Pizziolo (archeologa, referente Preistoria), Lucia Sarti (archeologa), Edoardo Vanni (archeologo, referente Età classica), Nicoletta Volante (archeologa). In particolare l'equipe del progetto TraTTo che ha svolto la ricognizione archeologica nell'ottobre 2016 all'interno del Parco Regionale della Maremma è costituita da: Nicoletta Volante (responsabile scientifico); Giovanna Pizziolo (coordinamento e pianificazione); Michele De Silva, Davide Cristoferi, alle attività hanno partecipato collaboratori (Laura Morabito) e alcuni studenti del Dipartimento di Scienze Storiche e del Beni Culturali afferenti ai Corso di Laurea in Scienze storiche e del patrimonio culturale, del Corso di Laurea Magistrale in Archeologia e del Master di II livello in Geotecnologie per l'Archeologia dell'Università di Siena.

Il presente lavoro è frutto della piena collaborazione tra gli Autori: si devono comunque imputare a N. Volante il capitolo «Fonte e metodi: i dati archeologici» (\$5-7), a

D. Cristoferi il capitolo «Fonte e metodi: i dati storici» (\$8-11), a M. De Silva il 
capitolo «Banche dati e GIS» (\$12-28), a G. Pizziolo il capitolo «La ricognizione archeologica» (\$29-51); a tutti gli Autori i capitoli «Introduzione» e «Osservazioni finali».

\section{Introduzione}

1 Il progetto TraTTo (Transumanza e Territorio in Toscana) nasce nell'anno accademico 2014-2015 dalla collaborazione fra il Dipartimento di Scienze Storiche e dei Beni Culturali dell'Università degli Studi di Siena e l'École française de Rome nell'ambito del Programme scientifique 2012-2016 La transhumance en Italie centrale de la Protohistoire à nos jours (Axe 1. Parcours et échanges en Méditerranée; Thème 1. Espace économique), coordinato da Stéphane Bourdin (directeur des études pour l'Antiquité, École française de Rome ) e Saverio Russo (Università degli Studi di Foggia) ${ }^{1}$.

2 La collaborazione, tramite una convenzione e il finanziamento delle attività da parte dei due istituti, ha come scopo lo sviluppo di ricerche interdisciplinari per la ricostruzione e l'analisi delle pratiche transumanti nell'Italia centrale, in particolare in Toscana, nella lunga durata, dalla Pre e Protostoria all'Età contemporanea (III millennio a.C.-metà del sec. XX d.C.). Il progetto ha visto la partecipazione di archeologi, storici e geografi del Dipartimento con i seguenti obiettivi: a) identificare e georeferenziare percorsi e pascoli legati alle pratiche transumanti in Toscana nella lunga durata; $b$ ) identificare e testare metodologie interdisciplinari di tipo qualitativo, quantitativo e di analisi regressiva per lo studio delle pratiche pastorali; c) verificare la continuità e discontinuità di uso dei percorsi e dei pascoli e i loro fattori di influenza, in particolare per il periodo premedievale. In questo modo si ritiene possibile ricostruire l'evoluzione delle attività pastorali nella lunga durata, dal pastoralismo mobile alla transumanza su vasta scala. Il progetto trae ispirazione dal metodo regressivo già sviluppato da Bloch per le campagne francesi, dal concetto di longue durée elaborato da Braudel per l'ambito storico e sviluppato da Barker per quello archeologico, così come dai più recenti sviluppi della landscape archaeology e dell'analisi di mobilità attraverso analisi predittive e postdittive anche nell'ambito toscano ${ }^{2}$.

3 Il lavoro di ricerca del biennio 2015-2016 si è concentrato sulla Toscana meridionale, in particolare sul comprensorio Monte Amiata-Maremma, all'interno dell'attuale provincia di Grosseto. La scelta è stata dettata dalla necessità di mettere a punto le metodologie in un'area limitata e allo stesso tempo dalle buone potenzialità. L'area maremmanoamiatina è un contesto ambientale dall'antica e forte tradizione pastorale, punto di arrivo invernale dei flussi transumanti dagli Appennini in età storica ma anche spazio autonomo per la mobilità pastorale di medio e corto raggio dal Monte Amiata alle piane costiere. Ad essa è riferibile una ricca bibliografia storica, archeologica e geografica, oltre ad un abbondante materiale di archivio per le pratiche transumanti di età tardo-medievale e moderna, dovuto alla presenza della Dogana dei Paschi di Siena ${ }^{3}$.

Le azioni di ricerca svolte nell'area ed oggetto del presente contributo sono state la costruzione del sistema informativo e la ricognizione archeologica nell'area del Parco Regionale della Maremma presso Alberese (GR). In primo luogo è stata svolta un'ampia ricognizione della bibliografia storica, archeologica e geografica alla scopo di raccogliere $i$ dati editi a disposizione sulle pratiche transumanti nella lunga durata per il territorio maremmano-amiatino. Inoltre sono stati consultati i risultati di due tesi di dottorato di ricerca in ambito storico e archeologico sulla transumanza per la medesima area e sono 
state svolte ulteriori ricerche sulla documentazione inedita negli Archivi di Stato di Siena e Firenze $e^{4}$. Le caratteristiche delle diverse tipologie di dati e le problematiche relative al loro inserimento nel database e al dialogo con le altre fonti sono trattati nella sezione Fonti e Metodi. La progettazione e la costruzione della piattaforma e della banca dati del sistema informativo, sulla base dei dati a disposizione, è descritta nella sezione Banche dati $e$ GIS, mentre la ricognizione archeologica è trattata nella sezione Ricognizione archeologica. Seguono alcune Osservazioni finali.

\section{Fonti e metodi: i dati archeologici}

5 La base informativa del progetto TraTTo e del sistema GIS è costituita dall'insieme dei dati storici e archeologici riconducibili alle attività di transumanza o in senso più generale alle attività di allevamento legate alla mobilità di breve, medio e lungo raggio. In via generale, in questa prima fase le operazioni si sono concentrate su attività di censimento e acquisizione dati di varie tipologie e ambiti cronologici. La nostra attenzione è stata dedicata a dati di tipo puntuale riferibili a evidenze archeologiche o storiche relative a percorsi, strutture, siti, o semplici elementi di cultura materiale provenienti da censimenti bibliografici, da banche dati o da indagini svolte sul campo da membri del gruppo di lavoro del progetto TraTTo.

6 Il progetto prevede di inserire i dati provenienti da indagini stratigrafiche che possano essere relazionati ad attività di allevamento e a spostamenti sul territorio interpretabili come mobilità stagionale riconducibile alla transumanza. Infatti, specialmente per le fasi più remote del popolamento olocenico non è possibile individuare nettamente quali evidenze siano da imputare in modo circostanziato alle attività di transumanza rispetto a quelle relative a forme di sussistenza legate ad economie di allevamento. Anzi, il problema dell'identificazione di marcatori e tipologie di evidenze archeologiche e paleoambientali ${ }^{5} \mathrm{da}$ collegare alle attività di transumanza è una questione aperta ${ }^{6}$ alla quale con il progetto TraTTo vorremmo cercare di rispondere almeno in parte. A tale discussione si aggiunge di conseguenza la problematica relativa alla individuazione di un limite cronologico al quale riferire l'inizio delle modalità di allevamento transumanti.

7 In base a queste considerazioni l'impostazione del progetto ha previsto l'acquisizione delle evidenze edite riferibili a siti neolitici, eneolitici e dell'età del Bronzo facendo riferimento a iniziative di catalogazione e studi di settore ${ }^{7}$ presenti in letteratura o portate avanti attraverso la redazione di cartografia archeologica. Ovviamente l'esame dei notiziari della Soprintendenza Archeologica della Toscana e delle riviste di settore ha costituito una base di partenza per il censimento dei siti noti. L'acquisizione è stata dedicata a tutti i tipi di evidenze archeologiche ponendo particolare attenzione a quelle riferibili a strutture ed attività temporanee o stagionali così come le informazioni derivate dalle analisi paleo-ambientali relative ad aree di frequentazione. Inoltre, fra gli indicatori probabilmente legati alle attività di transumanza, la presenza del sale costituisce un elemento di estremo interesse che abbiamo cercato di censire attraverso l'acquisizione di evidenze perlopiù indirette ${ }^{8}$. Altrettanto interessante appare il legame tra economia pastorale in concomitanza con attività di carattere minerario e/o metallurgico, soprattutto per quanto riguarda le fasi iniziali della metallurgia in Europa ${ }^{9} \mathrm{e}$ nella penisola italiana. Proprio nell'Italia centrale tirrenica, nello specifico nella Toscana interna e costiera sembra possibile riconoscere un modello economico in cui pastorizia e metallurgia stabiliscono un rapporto sinergico ${ }^{10}$. Markers produttivi legati al sale e ai 
metalli potrebbero risultare indici indiretti di pastorizia transumante e come tali sono in corso di valutazione, a fronte di dati fortemente carenti e difficilmente interpretabili. Lo stesso approccio è stato adottato per il censimento delle evidenze riferibili all'età del Ferro, alla fase etrusca e romana. Per queste fasi le testimonianze archeologiche si arricchiscono anche di riferimenti tratti dalle indicazioni epigrafiche ${ }^{11}$ e dalle diverse forme di ritualità ad esempio connesse con il culto di Hercules, Diana e Selvans/Silvanus plausibilmente collegabili alla transumanza.

\section{Fonti e metodi: i dati storici}

8 I dati sulle pratiche, i percorsi e i pascoli transumanti desumibili dalla documentazione di archivio di età medievale e moderna raccolti nel corso dell'attività di ricerca negli Archivi di Stato di Siena e di Firenze o ricavati dalla bibliografia storica possono essere distinti, a seconda delle fonti di provenienza, in due principali tipologie, da cui derivano caratteristiche e limiti: quelli ottenuti da scritture private e quelli da documentazione pubblica.

9 I primi sono atti di compravendita di bestiame $o$, più spesso, di soccida. Quest'ultimo è l'affidamento del bestiame da un proprietario ad un pastore con la condivisione del rischio (perdita degli animali, furto, epizoozie) e dei proventi (prodotti, animali, ricavi dalla vendita) alla scadenza del contratto, secondo percentuali variabili. Sia gli atti di compravendita che quelli di soccida sono difficili da reperire nei registri notarili, data la natura di tali contrattazioni, spesso orali o dalla durata limitata nel tempo, oltre ai riferimenti non sistematici alle pratiche transumanti, quando queste avvenivano. Si tratta dunque di informazioni puntuali ed episodiche: quando vi sono, è possibile identificare l'area di provenienza del bestiame, quella di destinazione e il luogo in cui si svolge l'affidamento del bestiame, quando coincidono con quelle del proprietario o del soccidario, oltre che la composizione del gregge e il suo valore economico ${ }^{12}$. Maggiori informazioni sono estraibili dalla documentazione dei grandi enti monastici toscani, proprietari di greggi e pascoli dagli Appennini alla Maremma. Tale documentazione è spesso non seriale e incompleta, ma vi si possono trovare indicazioni maggiormente precise ed estese nel tempo sulle aree di pascolo o di sosta, le tecniche di allevamento, l'entità dei greggi. Si tratta di serie documentarie che si estendono maggiormente per l'Età moderna ${ }^{13}$.

10 Le fonti pubbliche sono costituite dagli statuti delle comunità rurali (XIII-XVIII secolo), la legislazione del Comune e poi dello Stato di Siena (XIII-XVIII secolo), quella della Dogana dei Paschi di Siena (1419-1778), i testi delle Visite compiute dagli ufficiali del Granduca di Toscana in Maremma (XVI-XVIII secolo). Si tratta di documentazione in buona parte conservata nell'Archivio di Stato di Siena. Gli statuti delle comunità maremmane offrono preziosi riferimenti, seppur puntuali nel tempo, sulle aree di pascolo collettivo e privato, sulle norme a protezione di queste superfici, sui punti di pedaggio del bestiame transumante ${ }^{14}$. Si tratta di informazioni che devono essere incrociate con quelle provenienti dalla legislazione del Comune di Siena. Dal XIII-XIV secolo esso costituisce il principale attore politico in Maremma, sostituendosi alle signorie locali e ai conti Aldobrandeschi e incamerando numerosi diritti di uso e proprietà di pascoli. Questi, a partire dal 1353 saranno riorganizzati nella Dogana dei Paschi di Siena, un'istituzione pubblica deputata alla gestione dei pascoli per il bestiame transumante in cambio del pagamento di una serie di pedaggi. Il comune di Siena prima e la Dogana poi, fino a tutta 
l'età moderna, hanno prodotto e conservato una vasta massa documentaria riguardante l'amministrazione dei pascoli maremmani, la definizione dei loro diritti di uso, l'identificazione del bestiame transumante, la gestione dei percorsi, l'esazione dei pedaggi e delle ammende per le infrazioni compiute dai pastori. Si tratta spesso di serie documentaria ampie, dettagliate ed estese nel tempo, specialmente per l'Età moderna, cui si aggiungono numerose petizioni, precise ma maggiormente puntuali, di comunità o pastori transumanti alle istituzioni senesi ${ }^{15}$. Le Visite Granducali, infine, offrono un'ulteriore prospettiva, seppur focalizzata sulle comunità rurali visitate con accuratezza e precisione dagli ufficiali del Granducato di Toscana e descritte minuziosamente nelle relazioni ufficiali che erano tenuti a riportare dai loro viaggi. Qui si possono trovare dati sull'estensione, la toponomastica e la capacità dei pascoli comunitari spesso utilizzati dai pastori transumanti, così come sull'entità delle mandrie di bestiame locale e sulle loro relazioni con la Dogana senese ${ }^{16}$.

11 In conclusione, si tratta spesso di informazioni puntuali, sovrapponibili solo parzialmente, ma che sono in grado di integrare altre tipologie di dati attraverso il metodo regressivo e la comparazione delle medesime aree nel tempo.

\section{Banche dati e GIS}

12 Gli studi sulla transumanza e più in generale sulle attività antropiche legate alla pastorizia hanno evidenziato con crescente enfasi negli ultimi anni la necessità di affrontare questo tema nella sua complessità storica e geografica ${ }^{17}$. Gli studi recenti segnano infatti un interesse sempre maggiore verso quegli strumenti informatici che consentono di trattare in modo complessivo dati provenienti da diverse fonti e ambiti disciplinari nella loro componente territoriale ${ }^{18}$.

13 Nel GIS del progetto TraTTo, dal punto di vista strutturale, possono essere distinti tre gruppi di strati informativi:

14 - le banche dati più generiche, che possiamo definire di contesto, come ad esempio la cartografia topografica e tematica generale (geologia, uso del suolo, DTM, etc.) e le ortofoto;

- le banche dati (esistenti o realizzate ad hoc) su tematiche più specifiche come i siti archeologici, la viabilità storica, la cartografia storica, la documentazione delle attività di ricognizione e di scavo, etc.;

- il geodatabase dei percorsi e dei luoghi della transumanza, che costituisce la sintesi delle attività di ricerca del progetto TraTTo, dove confluiscono le informazioni e le attestazioni specificamente attinenti alle tematiche del progetto.

15 Come è noto, una parte fondamentale della costruzione di un GIS consiste nell'acquisizione e organizzazione all'interno del sistema dei dati cartografici, aerofotografici, e tematici al fine di inquadrare le caratteristiche del territorio, analizzarne le relazioni e al contempo sviluppare la contestualizzazione dei dati antropici nella loro sedimentazione storica. Per quanto riguarda il progetto TraTTo, si sono utilizzate diverse banche dati (di contesto generale o su tematiche più specifiche) disponibili presso i Laboratori del Dipartimento di Scienze Storiche e dei Beni Culturali dell'Università di Siena. Inoltre, sono state utilizzate le connessioni WMS con le banche dati messe a disposizione dalla Regione Toscana ${ }^{19} \mathrm{e}$ dal Geoportale Nazionale ${ }^{20}$. 
16 Di particolare efficacia ai fini della ricerca è risultato l'uso delle foto aeree in bianco e nero risalenti agli anni cinquanta e settanta del secolo scorso, ortorettificate, mosaicate e rese disponibili dalla Regione Toscana. In particolare, la fonte più interessante e ricca di spunti interpretativi è risultata la ripresa effettuata nel 1954 dal volo GAI. Queste fotografie aeree infatti ritraggono il territorio toscano prima delle grandi trasformazioni avvenute dopo il boom degli anni sessanta; la loro consultazione ci consente quindi di apprezzare nelle aree pianeggianti un contesto rurale non ancora compromesso dall'espansione dei centri abitati e dalla creazione delle infrastrutture (si veda ad es. l'area intorno a Grosseto) e nelle aree collinari e montane possiamo osservare un uso del suolo che rispecchia i caratteri tradizionali del paesaggio toscano e soprattutto, se le fotografie sono ad una scala adeguata, possiamo evidenziare la presenza di una rete di comunicazioni e assi viari minori riconducibili a percorsi pedestri o per animali oggi ormai in disuso o abbandonati.

17 Un capitolo a sé stante è rappresentato dalla cartografia storica. La possibilità di inserirla nel GIS dipende da molte variabili che mutano da caso a caso. Tuttavia, in riferimento alla cartografia storica, sono di particolare rilievo icatasti storici anche per le sperimentazioni fatte per georeferenziarli. In questo panorama, un felice esempio, particolarmente importante per la sua valenza storica e il suo ricchissimo contenuto informativo, è costituito in Toscana dal Catasto Leopoldino (realizzato fra 1817 e il 1835) sul quale, anche all'interno del Dipartimento di Scienze Storiche e dei Beni Culturali sono stati svolti numerosi studi, e sviluppate le procedure di georeferenziazione ${ }^{21}$. Le mappe del Catasto Leopoldino, insieme a quelle dei catasti ottocenteschi del Ducato di Lucca e del Ducato di Massa e Carrara, sono ad oggi disponibili per la consultazione in rete, anche via WMS, attraverso il progetto CaStoRe ${ }^{22}$ (Catasti Storici Regionali). Il Catasto Leopoldino, acquisito in WMS all'interno del GIS del progetto TraTTo, costituisce una fonte fondamentale per sviluppare la ricerca: da una parte infatti ci permette di verificare alcune informazioni storiche derivate dalle analisi archivistiche e dallo studio delle evidenze archeologiche, dall'altra costituisce di per se una fonte storica da analizzare per mettere in evidenza elementi della toponomastica, della viabilità e dell'uso del suolo riconducibili a pratiche ottocentesche legate alla transumanza. Tali informazioni, oltre a rivestire un interesse di per se come fonte storica del primo Ottocento, sono talvolta riferibili anche a periodi precedenti. Il catasto rappresenta infatti una fonte insostituibile per indagare la memoria dei luoghi e delle attività pastorali itineranti sia a breve che $\mathrm{a}$ lungo raggio.

18 Un'altra tipologia di dati deriva dalle analisi cartografiche e storiche presenti nel fondamentale testo di riferimento «I percorsi della transumanza in Toscana» ${ }^{23}$ che offre un ampio inquadramento del fenomeno storico, dell'età moderna e contemporanea, toccando anche aspetti etno-antropologici e propone un'attenta e dettagliata ricostruzione dei percorsi individuati nelle loro connessioni come parte di una rete legata a varie forme di mobilità. Il testo presenta informazioni puntuali riferite a evidenze storiche e un ricco apparato cartografico dove gli Autori propongono una serie di tracciati riferibili alla transumanza suddivisi per diverse tipologie.

19 Si è pertanto ritenuto opportuno partire dall'acquisizione di queste informazioni in modo da avere a disposizione un corpus di dati relativi alle ultime fasi di uso di questa pratica di allevamento ma che sono potenzialmente riconducibili alle fasi precedenti. La rete dei percorsi della transumanza acquisita all'interno del GIS ci permette infatti di procedere a ritroso, adottando il metodo di analisi storico regressivo, e di ipotizzare continuità o 
trasformazioni nell'organizzazione delle modalità di allevamento e nelle scelte dei percorsi. In altre parole, l'acquisizione in forma di livelli vettoriali di queste informazioni all'interno del GIS ci permette di fare confronti e verifiche sulla sedimentazione di certe pratiche legate alla transumanza e di individuare tracce di antichi percorsi.

Da un punto di vista operativo abbiamo pertanto vettorializzato in forma di linee i tracciati della viabilità della transumanza riportando le specifiche presenti nella cartografia allegata ${ }^{24} \mathrm{e}$ nel testo rispetto a tutto il territorio toscano. Oltre ai tracciati viari abbiamo acquisito in forma di punti tutte le evidenze legate a strutture, luoghi di sosta, ospedali, dogane, valichi etc.; inoltre sono state acquisite le informazioni legate alla toponomastica che può rimandare alle attività di transumanza. Particolare attenzione è stata dedicata all'area di studio per la quale maggiori sono state le verifiche topografiche in fase di acquisizione.

21 Se le banche dati di contesto e di supporto all'analisi costituiscono il 'tavolo da lavoro' del progetto, è risultata subito evidente la necessità di costruire una banca dati che raccogliesse in forma organica e sintetica i risultati della ricerca, vale a dire tutte le attestazioni - e relative informazioni - di elementi riconducibili alle attività legate alla transumanza (o più genericamente alla mobilità, alla pastorizia e all'allevamento) che fossero emerse nel corso dell'indagine. Alla progettazione e strutturazione di questa banca dati dei percorsi e dei luoghi della transumanza, che costituisce il cuore del sistema ed il contributo originale del progetto, è stata rivolta una particolare attenzione.

In primo luogo è stata sviluppata una riflessione metodologica sulle caratteristiche che la banca dati ed il relativo sistema di gestione dovevano assumere per poter trattare informazioni così diversificate. Infatti il progetto prevede l'acquisizione, la gestione e l'analisi di una grande quantità di dati derivanti da diverse tipologie di fonti. Le informazioni - e gli oggetti ai quali queste si riferiscono - differiscono per tipologia (evidenze archeologiche, attestazioni storiche, toponomastiche, documentarie, etc.), per cronologia (che si estende dalle fasi pre e protostoriche all'età contemporanea) e per geometria (ovvero punti, linee e poligoni, ad esempio punti per evidenze circoscritte e sporadiche, linee per percorsi lineari, poligoni per aree di interesse o di uso del suolo). Tutto ciò implica, ancor prima di iniziare a raccogliere informazioni, la necessità di elaborare uno schema concettuale relativo alle entità coinvolte e alle loro reciproche relazioni. Tale schema costituisce l'indispensabile punto di partenza per la progettazione e la costruzione di un efficiente base dati in grado di rappresentare un fenomeno così complesso come quello della transumanza. In altre parole, dobbiamo immaginare un geodatabase (o meglio, uno spatial database) in grado di gestire informazioni relative ad entità complesse talvolta molto diverse fra loro, alla loro geometria (posizione, forma $\mathrm{e}$ topologia) ed alle fonti che ne attestano l'esistenza. Il sistema deve inoltre consentire l'immissione dati da parte di utenti diversi e deve per questo avere interfacce semplici e una struttura solida che regoli le relazioni fra dati di natura diversa.

In base ai criteri discussi con i colleghi del Dipartimento si sono individuate necessità specifiche relative all'organizzazione delle informazioni, alla loro compatibilità con altre banche dati istituzionali esistenti e alla possibilità di fare scelte operative che prevedessero l'uso di sistemi Open Source.

24 La struttura del geodatabase (fig. 1) è concettualmente costituita da tre entità principali relazionate fra loro:

- il sito, inteso come localizzazione fisica dell'evidenza, al quale possono essere riferite più attestazioni; 
- l'attestazione di un oggetto o di una attività legata alla transumanza derivante da una più fonti e riconducibile ad un sito;

- la fonte (bibliografica, documentaria, archeologica etc.) da cui deriva una o più attestazioni.

Fig. 1 - Trattamento dati: schema generale.

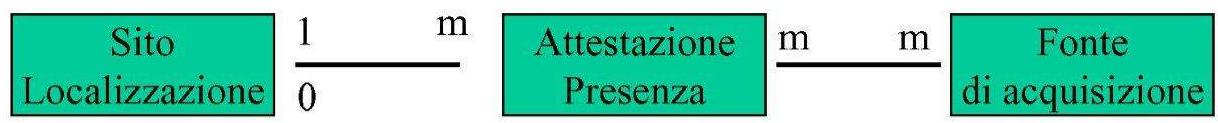

Per sito (luogo) si intende la localizzazione di uno o più oggetti o attività la cui presenza è attestata da più fonti.

Es: solo la "localizzazione" di un diaccio e di una dogana cha hanno diverse attestazioni su più fonti.
Attestazione della Fonte da cui abbiamo presenza di un oggetto o ricavato l'attestazione. attività derivata da una $\mathrm{o}$ Non fonte primaria. più fonti. L'attestazione potrebbe non avere una localizzazione.

Tabelle diverse per le varie tipologie di fonti (biblio, documento Una attestazione per ogni d'archivio, report di tipo di oggetto o scavo, DB). fenomeno (diaccio e dogana nello stesso luogo).

Tale schema concettuale è stato adottato per l'implementazione del database spaziale dei percorsi e dei luoghi della transumanza del progetto TraTTo, attraverso la realizzazione di una struttura relazionale più complessa nella quale ad ogni entità sono associate le informazioni ad essa pertinenti (fig. 2).

Fig. 2 - La struttura del database.

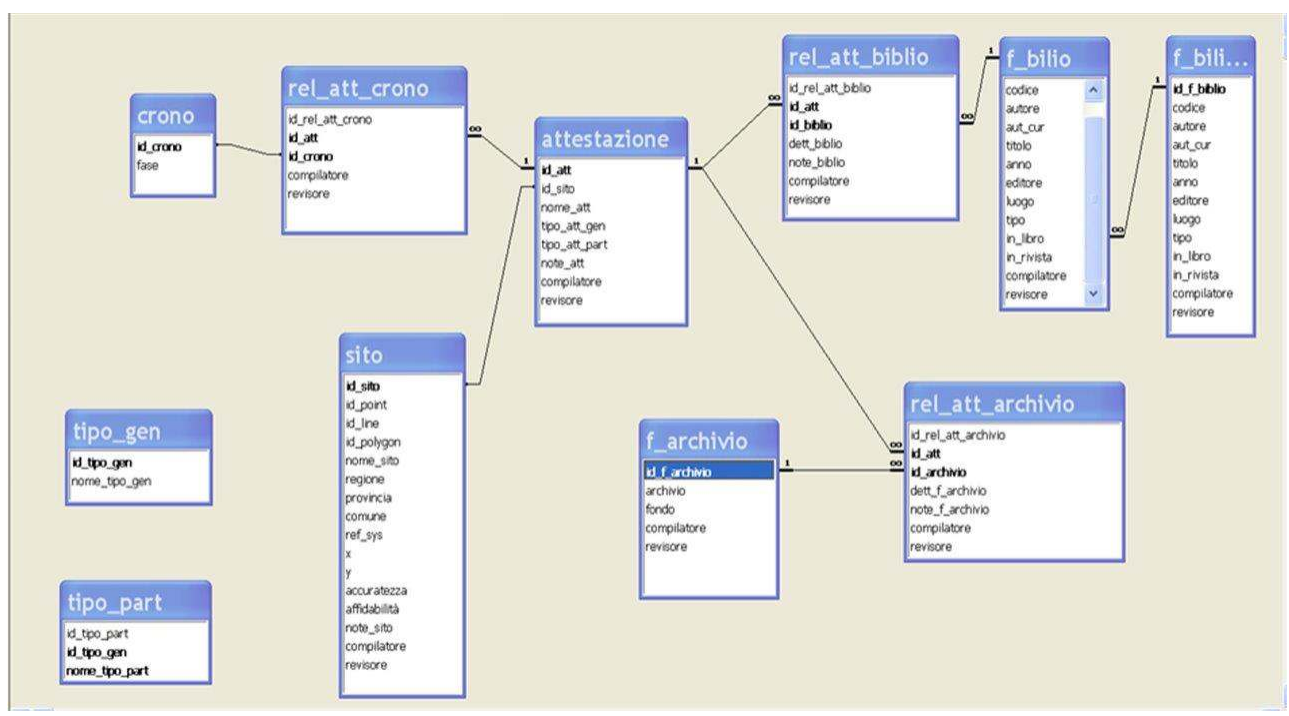

In sintesi, il GIS nel suo complesso è costituito dall'insieme delle banche dati che, strutturate in un ambiente condiviso, ci consentono di attivare processi di integrazione e di validazione delle informazioni, procedendo a scale e fonti integrate secondo la metodologia di lavoro propria della geografia storica. 

nell'area meridionale del Comune di Grosseto in accordo con la Soprintendenza ${ }^{25} \mathrm{e}$ ha usufruito dell'appoggio logistico delle strutture del Parco Regionale della Maremma. Alle attività di ricerca sul campo hanno partecipato alcuni componenti del gruppo di lavoro del progetto TraTTo e studenti e collaboratori del Dipartimento di Scienze Storiche e dei Beni Culturali dell'Università di Siena. ricognizione archeologica sistematica di alcune aree ad alto potenziale informativo e le verifiche a campione, in forma di sopralluoghi, di contesti specifici contraddistinti da elementi geomorfologici e toponomastici riferibili ad attività di allevamento o transumanza. Oltre alle indagini in oggetto si sono anche effettuate visite ai siti archeologici noti nell'area di studio al fine di incrementare con osservazioni dirette sul campo alcune informazioni relative al contesto geomorfologico e alle relazioni con il paesaggio del passato.

31 L'area compresa nel territorio grossetano è oggetto di indagini da parte del Dipartimento di Studi Storici e dei Ben Culturali da molti anni e ha visto ricerche svolte sia nell'ambito delle ricognizioni archeologiche sia con indagini stratigrafiche rivolte a contesti di diversa tipologia e riferibili ad un intervallo cronologico che va dal Paleolitico al Medioevo ${ }^{26}$.

32 All'interno di questo ambito di studi, la campagna di ricognizione svolta durante il mese di ottobre 2016 si focalizza per la prima volta sul tema della transumanza ponendosi obiettivi specifici per dare prime risposte alle questioni aperte presentate nei precedenti paragrafi.

33 Le indagini sul campo si configurano infatti come un primo test sulla capacità di intercettare in superficie le evidenze legate al fenomeno della transumanza. Si sono pertanto scelte alcune aree campione sia al fine di valutare potenzialità e prospettive del metodo di analisi adottato, che di procedere al contempo con l'acquisizione di informazioni di dettaglio da associare ai dati sulla transumanza già in nostro possesso.

L'area oggetto della ricognizione 2016 corrisponde alla sponda sinistra del fiume Ombrone che si estende dalla località Grancia a Marina di Alberese e ai Monti dell'Uccellina ed è in parte sotto la gestione e tutela del Parco Regionale della Maremma (fig. 3), questo territorio risponde positivamente ad alcuni criteri che abbiamo ritenuto fondamentali per effettuare un test efficace e affidabile. 
Fig. 3 - Inquadramento cartografico delle aree indagate.

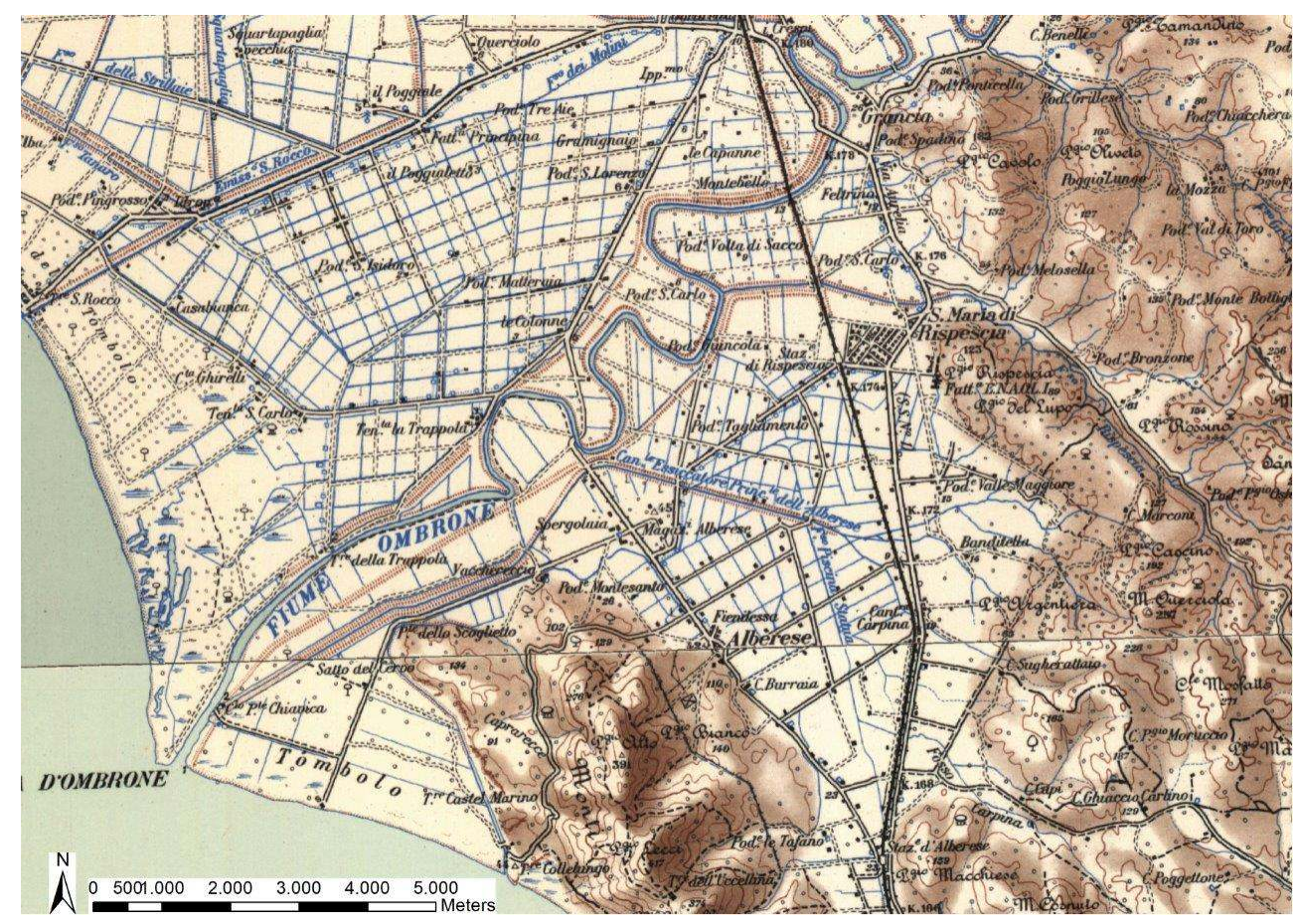

35 In sintesi la scelta della zona in cui svolgere le ricognizioni è stata orientata dalla presenza di alcune variabili che a nostro avviso possono influenzare il buon esito della ricerca. L'area delle indagini risponde positivamente a questi criteri:

a) presenza di evidenze archeologiche e storiche dalla Preistoria all'Età moderna: in una prospettiva di lunga durata diventa infatti importante indagare aree che mostrano una continuità di frequentazione attestata possibilmente in tutti i macro periodi in esame; in tal modo diventa più probabile tentare di rintracciare elementi di continuità della transumanza.

b) contesto territoriale conservativo a livello storico, archeologico e naturalistico: come è noto le indagini di superficie risentono negativamente dell'impatto antropico da imputare alle recenti attività di espansione dei contesti urbani e infrastrutturali; tali trasformazioni alterano negativamente anche la lettura del contesto ambientale del passato. Per aumentare le possibilità di individuare gli elementi che caratterizzano il paesaggio della transumanza in una prospettiva di lunga durata si è scelto il contesto del Parco Regionale della Maremma che fin dalla sua costituzione nel 1975 preserva il suo territorio da azioni antropiche con forte impatto di trasformazione dell'uso del suolo.

Oltre a queste caratteristiche che facilitano l'individuazione dei contesti con alto potenziale archeologico, abbiamo selezionato l'area di studio in base alla potenziale rilevanza per le attività transumanza. i criteri di selezione hanno riguardato le caratteristiche topografiche e morfologiche e le notizie storiche che testimoniano la presenza di questo fenomeno negli ultimi secoli. Di conseguenza sono state valutate positivamente le seguenti zone:

c) area che corrisponde al corridoio preferenziale della viabilità fra Maremma settentrionale e meridionale; si tratta di un passaggio in area pianeggiante compreso tra i Monti dell'Uccellina e le morfologie interne, è ancora ad oggi la zona dove si concentra la viabilità che connette la piana grossetana con le aree agli sbocchi delle valli del Fiora e dell'Albegna e in generale la zona costiera della Toscana meridionale e dell'alto Lazio. 
d) area che risulta il punto di arrivo dei flussi transumanti attestati in epoca medievale e moderna; le fonti storiche consultate indicano che la zona di Alberese ha costituito una delle aree terminali fra i percorsi di transumanza lungo le direttrici Maremma-aree interne della Toscana (fig. 4).

e) area storicamente interessata dallo sfruttamento di pasture invernali (bovini e ovicaprini); questo dato storico attesta, anche da un punto di vista ecologico, le buone condizioni dei pascoli di questa zona durante l'inverno. Possiamo supporre che queste caratteristiche si siano mantenute anche nel passato? Alcune risposte possono forse arrivare anche dalle verifiche sul campo.

Fig. 4 - Una ripresa aerea (2004) dell'area in esame. In rosa sono indicate le vie armentizie acquisite in forma di linee vettoriali dagli elaborati di Marcaccini, Calzolai 2003.

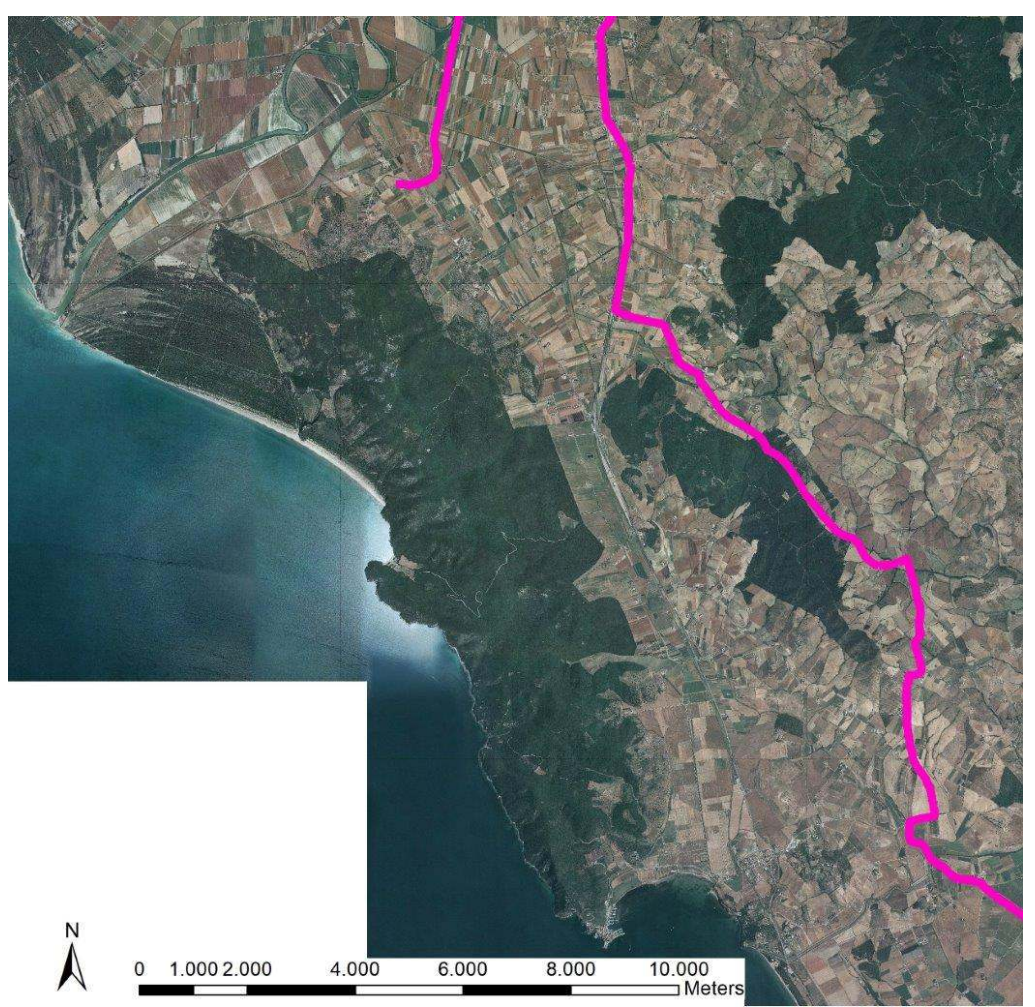

\section{La scelta delle unità di ricognizione}

Le attività di indagine sul campo sono avvenute attraverso l'individuazione delle Unità di Ricognizione, ovvero le particelle colturali all'interno delle quali si è svolta la ricognizione archeologica sistematica. Per questa prima fase di verifica i criteri di determinazione delle unità hanno mirato ad intercettare aree che avessero avuto una relazione diretta con elementi della viabilità storica, attestate o meno anche come vettori per spostamenti legati alla transumanza. In questo modo abbiamo voluto verificare le eventuali peculiarità o diversità offerte dal record archeologico da correlare alle attività di mobilità pastorale. Oltre a questo elemento, abbiamo ritenuto importante indagare altri fattori correlati alle attività di allevamento da rintracciare fra le caratteristiche topografiche e ambientali che potevano costituire motivi di interesse nelle strategie di mobilità e sosta. Per questo motivo sono state selezionate aree con particolarità morfologiche che potevano facilitare il raggruppamento del bestiame o il riparo. 
Da un punto di vista operativo, l'identificazione delle unità di ricognizione è stata basata sulle notizie storiche e archeologiche inserite nel GIS (vedi paragrafi precedenti) in particolare quelle relative agli elementi di viabilità. A queste indicazioni topografiche sono state affiancate le analisi delle morfologie (nello specifico svolte attraverso l'analisi del Modello Digitale del Terreno) e il confronto con le indicazioni desunte dall'analisi toponomastica.

Passando ad una scala di dettaglio, in pratica la scelta delle zone da ricognire e verificare, oltre a rispondere alle necessità di accessibilità e visibilità a terra, è stata basata su una serie di selezioni che hanno condotto alla identificazione delle seguenti zone:

- aree allo sbocco delle vie armentizie identificate da Marcaccini-Calzolai (2003) per l'Età moderna: versante sud-occidentale del Poggio di Magazzini di Alberese, aree prospicenti la Strada Provinciale di Alberese lungo il versante orientale del Poggio di Magazzini di Alberese (fig. 5).

- aree lungo la via Aurelia vetus di età romana e gli insediamenti prossimi alla stessa ${ }^{27}$ : area di Spergolaia.

- aree caratterizzate da elementi geomorfologici e toponomastici riferibili ad attività pastorali/transumanti: porzione NW dei Monti dell'Uccellina, località Mungitoio (pianoro a $65 \mathrm{~m}$ s.l.m. in prossimità di una piccola sella, attualmente coltivato ad oliveto con presenza di radure e aree di pascolo); fra i ripari naturali si evidenzia la «Grotta delle Caprarecce», articolata in vari ambienti e caratterizzata da un antro di ampie dimensioni, circa $45 \times 10 \mathrm{~m}$.

- aree caratterizzate da elementi geomorfologici e toponomastici con attestazioni documentarie di frequentazione transumante/pastorale ${ }^{28}$ : areale compreso tra le strade di Giuncola, Antica Dogana e Barbicato presso Rispescia; insediamento medievale di Poggio Cavolo/Montecalvoli (183 m s.l.m.); area tra la riva sinistra dell'Ombrone e la località Grancia, fattoria dell'Ospedale di S. Maria della Scala di Siena attivo in età medievale e moderna.

- aree con siti archeologici noti di varie epoche: aree lungo il Fiume Ombrone e aree lungo la paleofalesia detta «scogliera», in particolare in presenza di grotte naturali e ripari nel tratto tra lo Scoglietto e la torre di Collelungo.

Fig. 5 - La collina di Magazzini di Alberese e la fascia della pianura interessata dalla indagini.

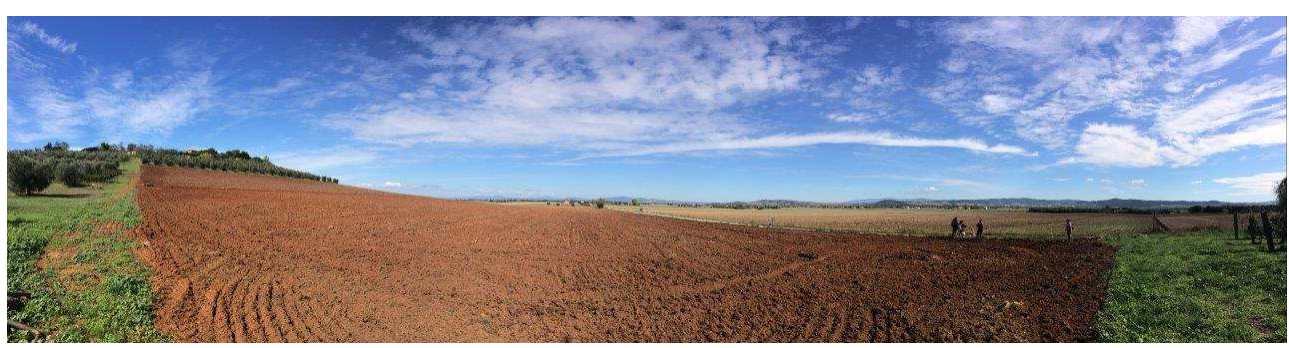

\section{Sintesi delle indagini}

Le attività si sono svolte nel settore settentrionale dei Monti dell'Uccellina, in particolare si sono concentrate lungo la fascia pedecollinare del versante NE investigando attraverso ricognizione sistematica unità colturali a seminativo nudo arate e zone arate o fresate all'interno di oliveti e vigneti (fig. 6). 
Fig. 6 - Attività di field survey in prossimità di Alberese.

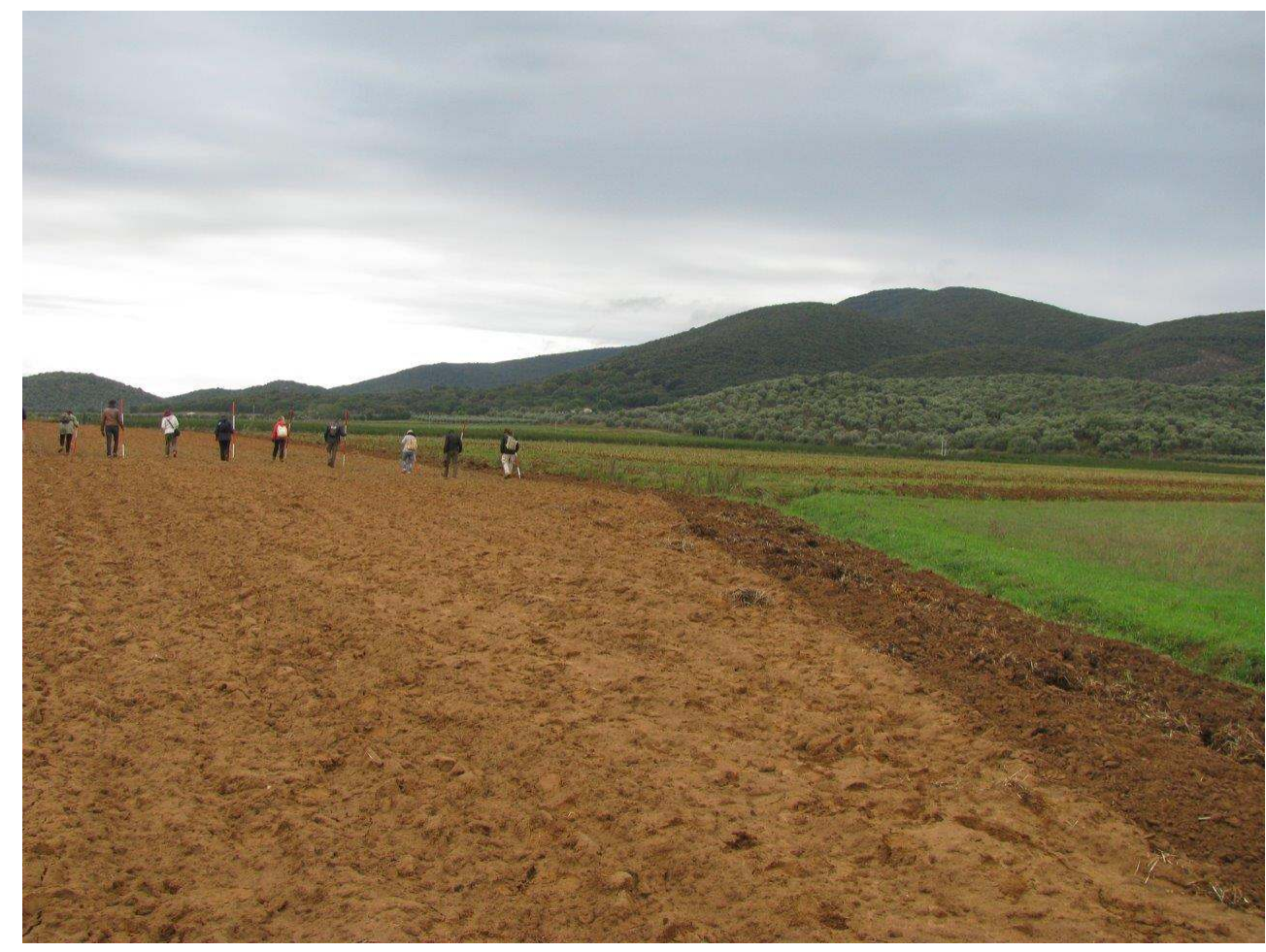

Particolare attenzione è stata anche dedicata all'area di Magazzini di Alberese (fig. 7), che è caratterizzata dalla presenza di una collinetta che si eleva nella zona pianeggiante compresa tra il centro abitato di Alberese e il fiume Ombrone. Si tratta di una porzione della piana alluvionale che è stata in parte fortemente modificata dalle azioni di bonifica che in questa porzione della pianura grossetana ha avuto luogo in vari momenti storici a partire dal XVIII sec. fino agli interventi della prima metà del secolo scorso. 
Fig. 7 - La distribuzione delle Unità di Ricognizione in prossimità di Magazzini di Alberese, sullo sfondo la fotografia aerea del 1954.

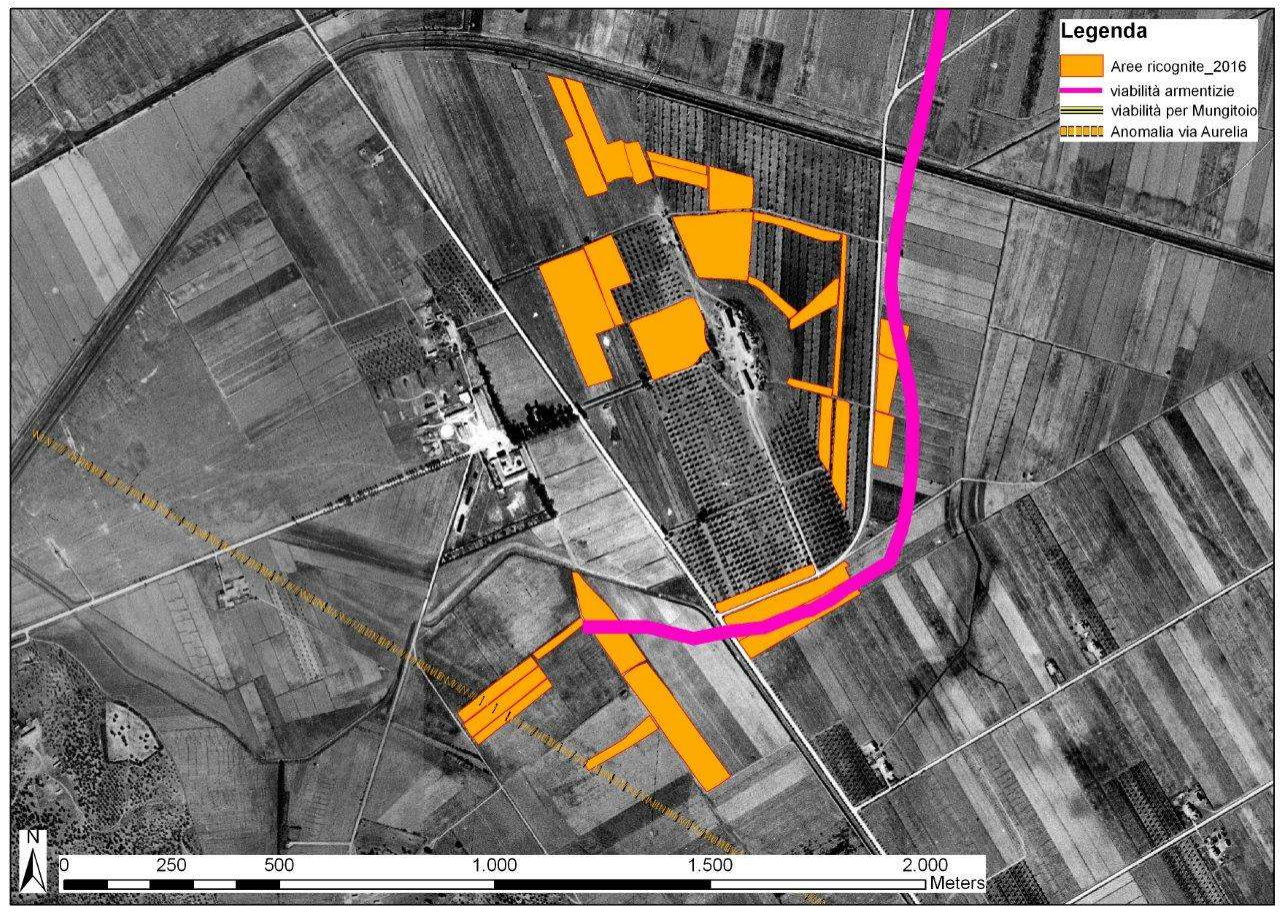

Per quanto riguarda le morfologie della porzione NW dei Monti dell'Uccellina, è stata dedicata particolare attenzione alla sella che collega la fascia pedecollinare compresa tra Alberese e la zona del Magazzini con l'area dei poggi interni e il versante NW dei Monti. L'area costituisce un passaggio naturale di collegamento fra le due zone ed è caratterizzata da una morfologia con pianori sub orizzontali (fig. 8). 
Fig. 8 - L'analisi degli aspetti morfologici. La piccola sella di passaggio fra i versanti, evidenziato dal tratteggio rosso, in località Mungitoio.

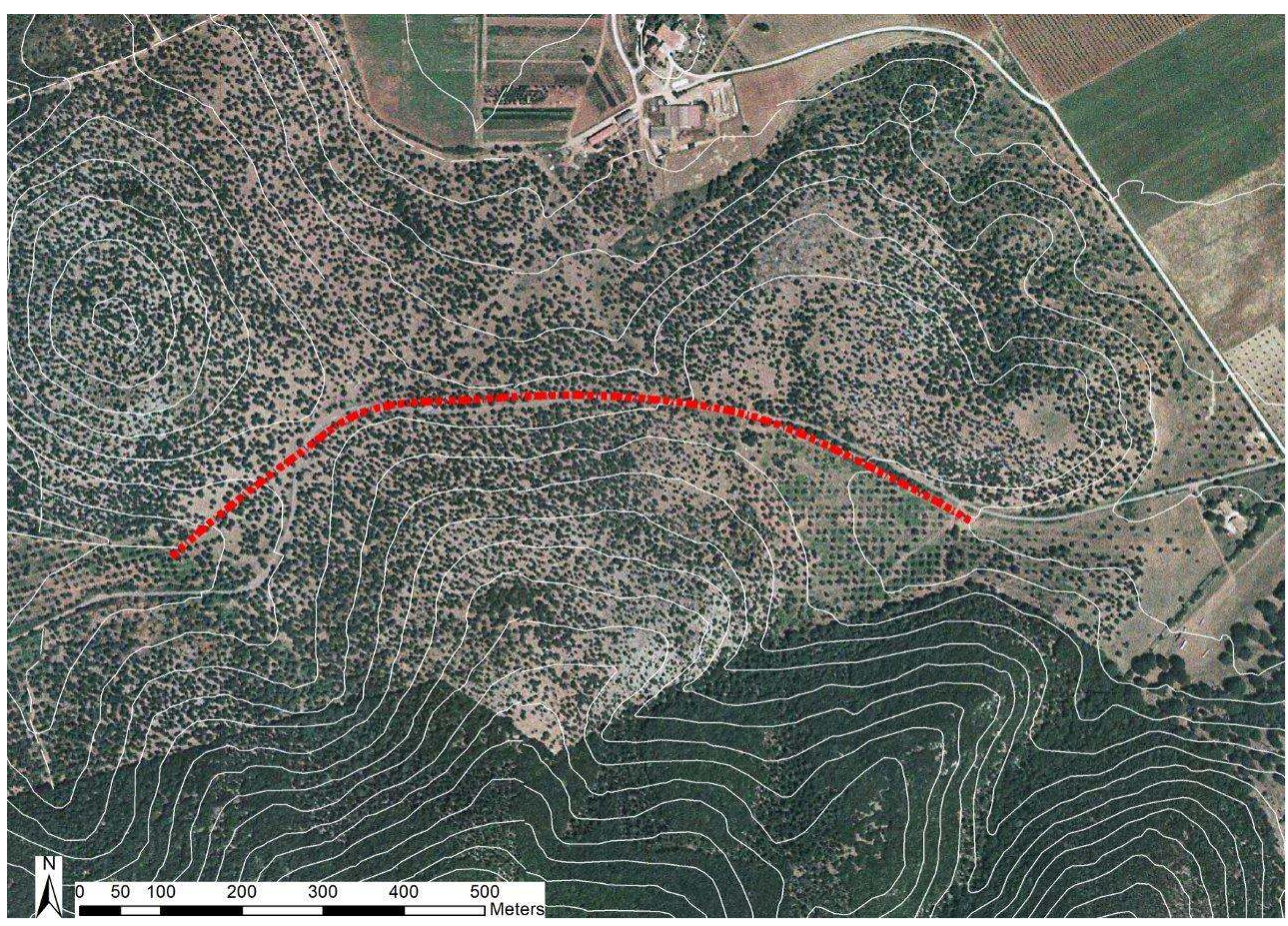

Le attività di ricognizione hanno individuato perlopiù manufatti riferibili a tutto l'arco cronologico in esame. Tuttavia in questo quadro si segnala la presenza più consistente di industria litica, in particolare relativa alle fasi oloceniche. La concentrazione del materiale in superficie ha permesso di delimitare aree di frequentazione più o meno intensa del territorio senza tuttavia identificare specifiche attività o aree funzionali.

L'acquisizione all'interno del GIS dei dati raccolti sul campo ha permesso di effettuare confronti con le basi informative: di particolare utilità risultano le analisi delle fotografie aeree attuali e storiche. Le immagini del 1954 restituiscono infatti gli assetti del paesaggio precedenti alle moderne attività agricole in cui l'uso ripetuto di mezzi meccanici e le conseguenti arature profonde hanno compromesso la leggibilità di alcune evidenze archeologiche in superficie (fig. 9). 
Fig. 9 - Le aree ricognite in prossimità di Alberese. La fotografia aerea del 1954 mette in evidenza alcune anomalie archeologiche, in particolare quelle relative alla viabilità romana.

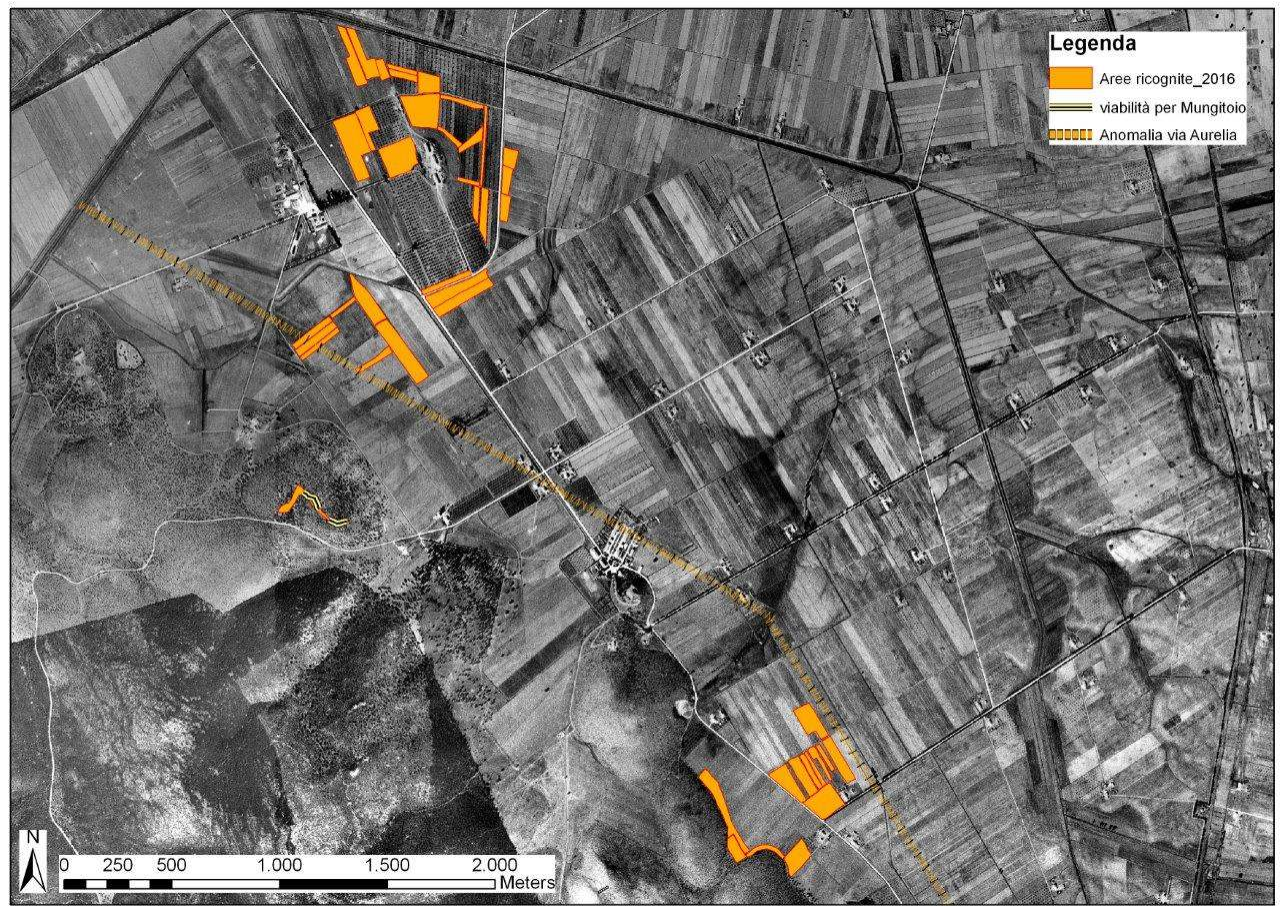

Infine, oltre alle ricognizioni sistematiche sono stati svolti sopralluoghi lungo la paleo falesia (fig. 10) nel tratto compreso tra le località Scoglietto e Collelungo con visita a grotte e ripari (ad esempio Grotta dello Scoglietto ${ }^{29}$ e Grotte delle Caprarecce) che testimoniano quanto la fascia della paleo falesia durante l'olocene abbia costituito un ambiente di forte interesse per l'uomo. Tuttavia è opportuno ricordare che le cavità e gli antri sono stati utilizzati per funzioni diverse nelle varie fasi di frequentazione. 
Fig. 10 - Una delle cavità che si aprono alla base della paleo falesia.

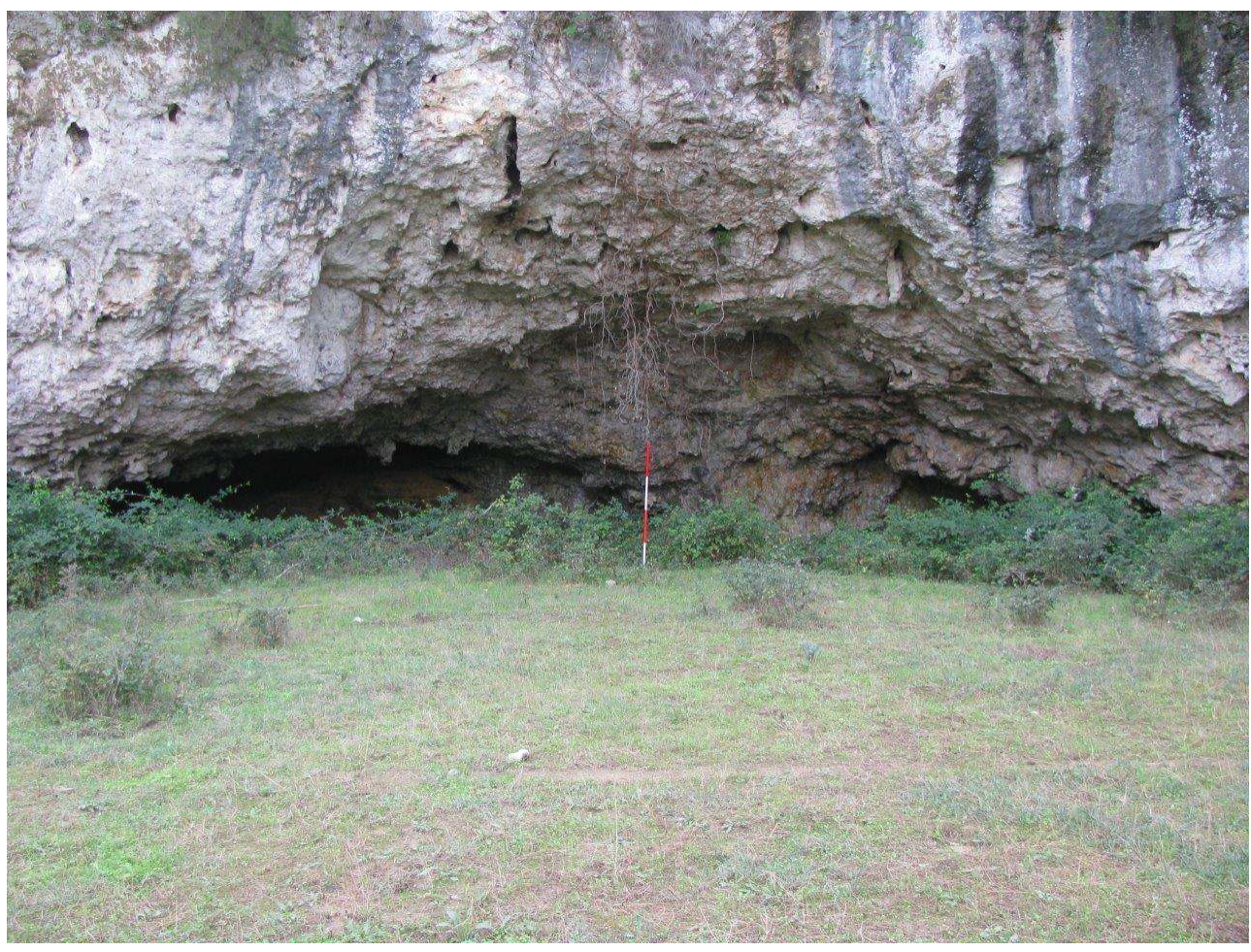

Altri tipi di sopralluogo si sono concentrati su evidenze toponomastiche e storiche anche al di fuori dei Monti dell'Uccellina. Di particolare interesse è risultato il sito di Poggio Cavolo (antico Montecalvoli / Monte o Poggio Cavolo) e del suo contesto topografico e la visita presso la Grancia (antica struttura agraria dell'ospedale di S. Maria della Scala di Siena).

\section{Risultati e criticità}

Come premesso, queste prime attività di ricognizione hanno avuto un duplice obiettivo: da una parte testare le potenzialità di ricerca dell'area in esame e dall'altra mettere in luce le criticità relative all'individuazione in superficie di evidenze archeologiche riferibili alle attività pastorali o di transumanza che generano plausibilmente strutture leggere e/o temporanee dalle caratteristiche effimere.

Esula dagli intenti di questo scritto presentare approfondimenti sul concetto di off site, ampiamente discusso in letteratura ${ }^{30}$. Vorremmo solo ricordare che resta ancora aperta la valutazione sulla quantità di manufatti sufficiente per l'individuazione di un cluster o una unità topografica, così come l'individuazione del loro limite spaziale, che variano notevolmente a seconda del contesto in analisi e del tipo di attività antropica che ha generato la formazione del deposito archeologico. Gli elementi di off site permettono infatti di ipotizzare aree di attività al di fuori degli insediamenti e queste possono essere testimoniate, ad esempio per le fasi della Preistoria olocenica, anche da pochi manufatti ${ }^{31}$. In questa prospettiva di lavoro si aprono diverse finestre interpretative per valutare le tracce lasciate da attività di tipo temporaneo, seppur ripetuto, o per attribuire un valore ad una diffusa presenza di materiale riferibile a diversi periodi e fasi storiche. 
49 Per quanto riguarda questo tipo di criticità, le ricognizioni sistematiche svolte fino ad ora per il progetto TraTTo hanno confermato la difficoltà nell'individuazione di tracce di strutture temporanee connesse ad attività pastorali dalla cronologia ben definibile. In generale le tracce di frequentazione antropica individuate nel corso di questa prima ricognizione sono difficilmente interpretabili da un punto di vista funzionale e mancano manufatti strettamente diagnostici connessi ad attività di produzione lattiero-casearia e tessile.

50 A questo dato si aggiunga la considerazione che l'intensa attività agricola, soprattutto a partire dalla metà del secolo scorso, ha molto probabilmente compromesso le evidenze di eventuali insediamenti temporanei connessi ad attività pastorali così come è accaduto in questa zona per alcune strutture insediative e per la viabilità romana.

51 In assenza di indicazioni storico-documentarie puntuali riferibili a strutture della transumanza nell'area pianeggiante, la ricognizione si è concentrata in aree che da un punto di vista pedologico risultavano non interessate da azioni di disturbo o copertura avvenute in tempi recenti. Il ritrovamento in superficie di manufatti preistorici in ossidiana, diaspro e selce riferibili all'olocene confermano l'ipotesi che tali suoli sono stabili e poco disturbati a partire almeno dal primo Olocene. La presenza di alcune concentrazioni di manufatti dovrà essere approfondita.

\section{Osservazioni finali}

52 Le attività di ricerca hanno visto una proficua collaborazione interdisciplinare che ha permesso di potenziare, anche durante le attività sul campo, il confronto di approcci e lo scambio di competenze archeologiche, storiche e geografiche all'interno del gruppo di lavoro.

53 Allo stesso tempo i sopralluoghi e le ricognizioni svolte hanno offerto alcuni spunti interessanti per quanto riguarda l'analisi globale dei dati grazie all'incrocio di informazioni di diversa tipologia. Ad esempio, l'area intorno all'insediamento collinare di Poggio Cavolo / Montecalvoli - fondato e frequentato fra X e XI secolo, riutilizzato secondo lo Statuto della Dogana dei Paschi del 1419 come area di controllo e conta del bestiame transumante per l'ampia visuale verso sud - ha mantenuto due toponimi pastorali riferibili alla transumanza (Caprarecce e Mandrioli) e risulta ancora oggi interessata dall'allevamento di ovini e cavalli fin sulla sommità.

54 Nell'area di Giuncola, lungo la riva sinistra dell'Ombrone, al termine di un'ansa del fiume, partiva secondo il Marcaccini-Calzolai (2003) la via di Dogana verso Alberese, che terminava tra Magazzini e Spergolaia. Osservando le foto aeree del volo GAI del 1954 è possibile notare un colore più chiaro delle acque fluviali, proprio in corrispondenza del punto di partenza della via di Dogana, questo suggerisce la possibile presenza di un guado sull'Ombrone (fig. 11). Si tratta di un'ipotesi da verificare in quanto il livello delle acque dell'Ombrone in regime di piena nei giorni della ricognizione non ha consentito ulteriori approfondimenti. 
Fig. 11 - Possibile guado lungo il Fiume Ombrone evidenziato nella fotografia aerea del 1954.

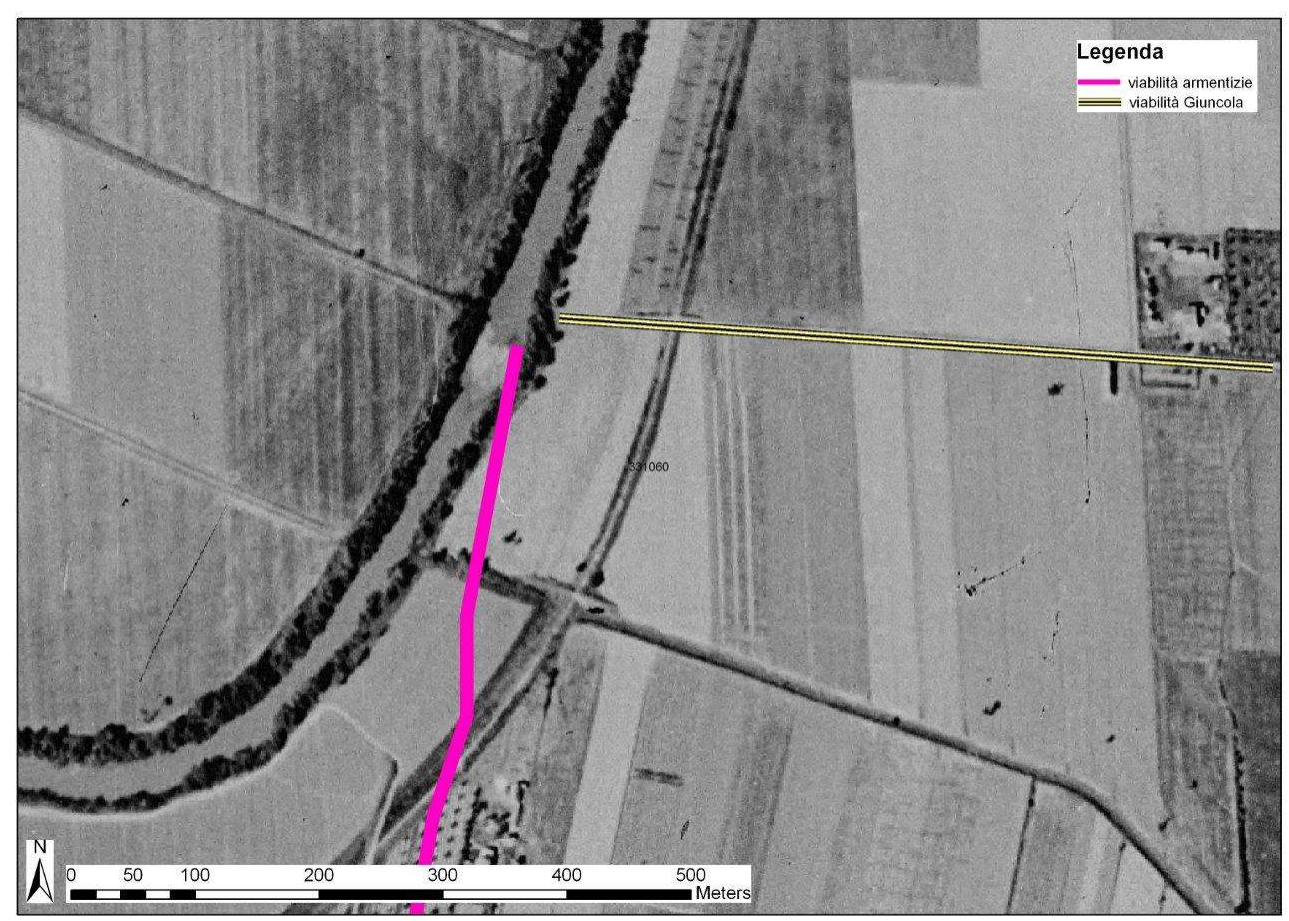

55 L'area del Mungitoio contiene altrettanti elementi di interesse: il toponimo infatti è legato ad un pianoro che si apre verso nord nei pressi di una piccola sella (65 s.l.m.) dei Monti dell'Uccellina. Il pianoro è dotato di alcune ampie radure mentre all'interno dell'oliveto storico che lo copre è praticato ancora il pascolo del bestiame bovino (fig. 12). L'area è lambita da una mulattiera ad oggi riconoscibile a tratti che sembra collegare i versanti dei Monti dell'Uccellina con la piana di Alberese (fig. 13).

Fig. 12 - Panoramica dell'oliveto presso la località Mungitoio.

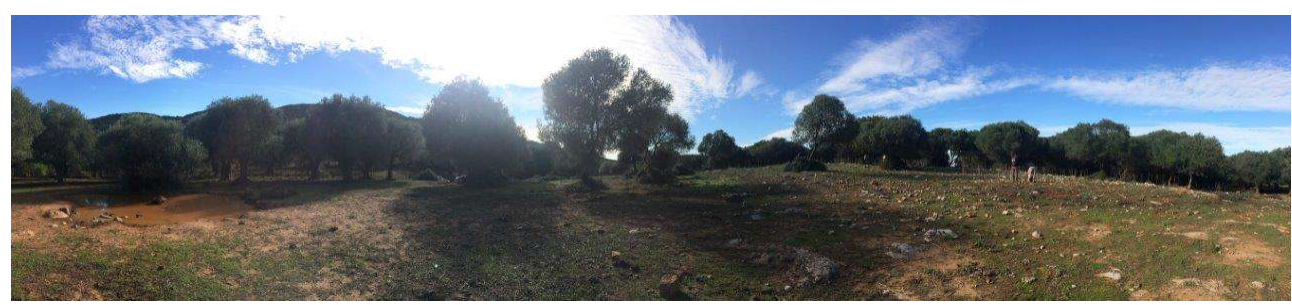


Fig. 13 - Evidenze relative alla viabilità ausiliaria in località Mungitoio.

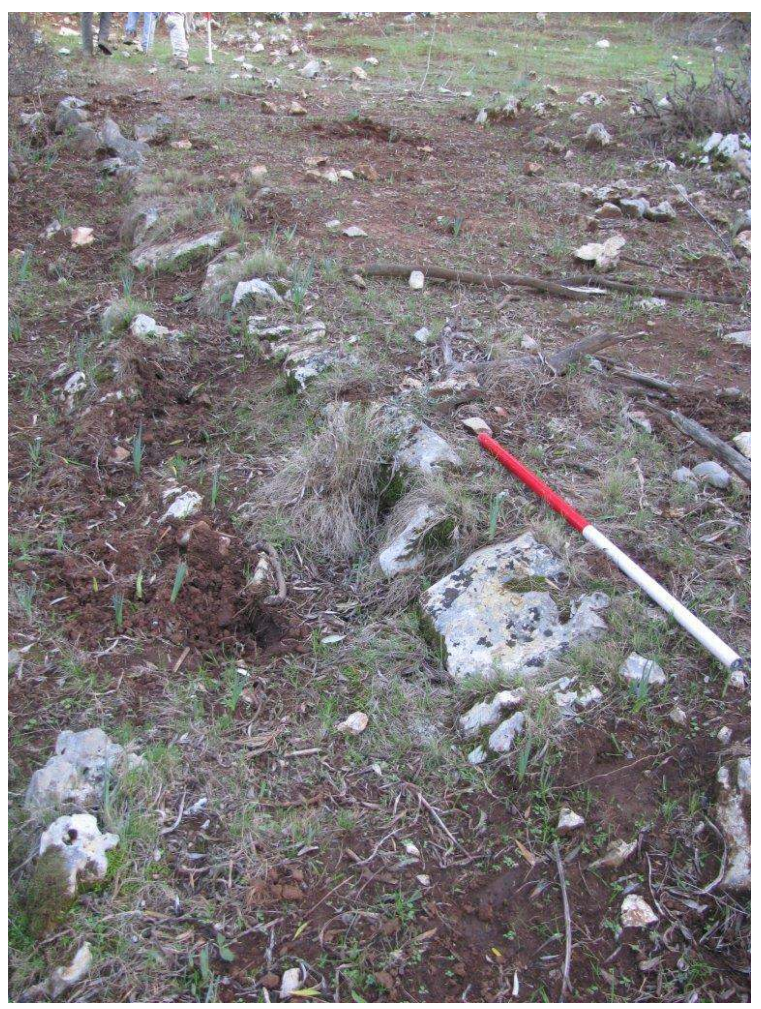

Nel Parco regionale si trova infine un sistema di grotte alla base della falesia che da Scoglietto va alla Torre di Collelungo e si affaccia su un'area pianeggiante formatasi in età storica oggi occupata da macchia mediterranea e pineta. La grotta principale di questo sistema, caratterizzata da un ampio ambiente $(45 \times 10 \mathrm{~m})$, dal nome di Caprarecce, potrebbe essere collegabile ad attività pastorali transumanti e stabili, praticate nei terreni dell'antica tenuta di Alberese in età storica (fig. 14). 
Fig. 14 - Uno scorcio dell'interno della Grotta delle Caprarecce.

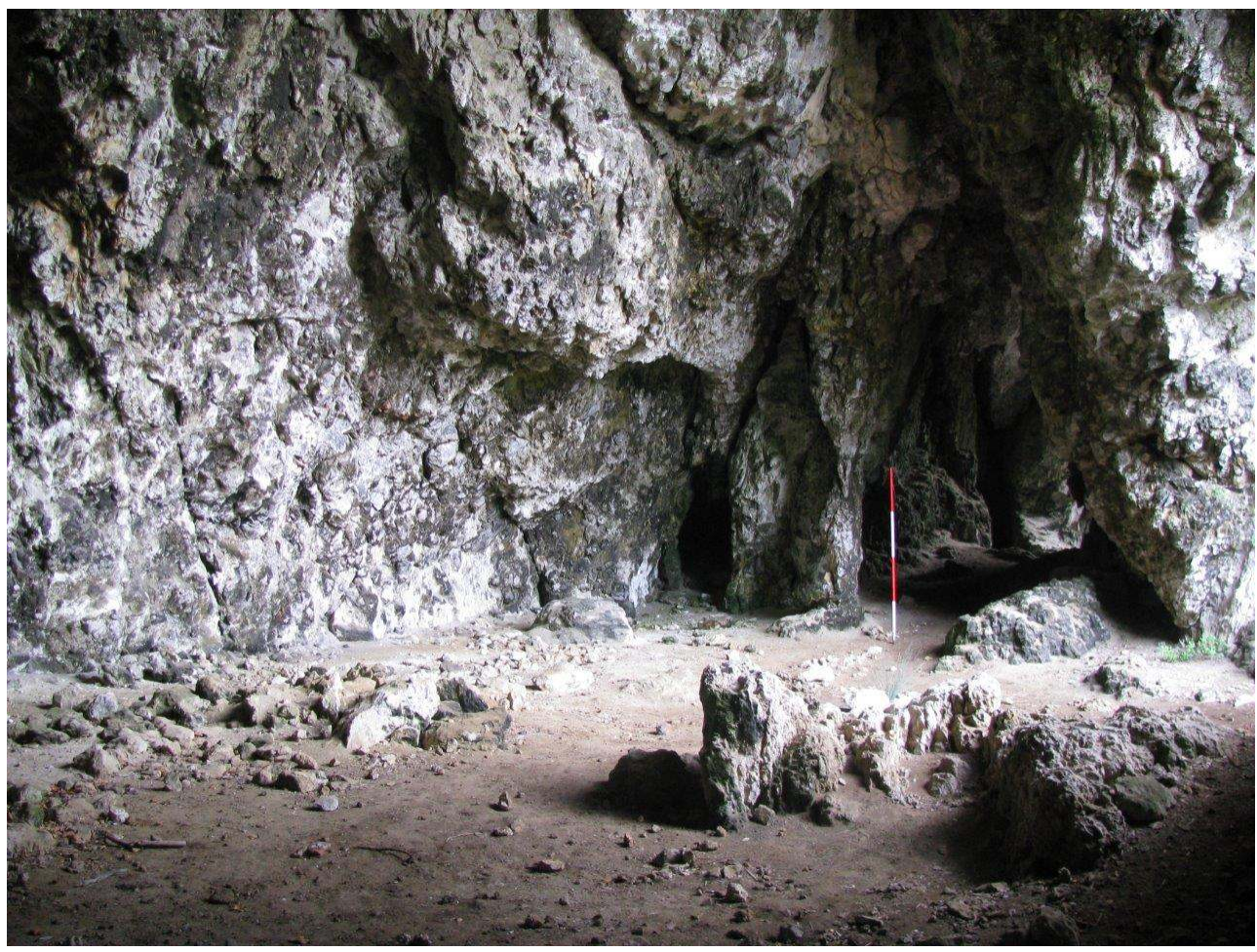

57 I dati emersi dalle ricognizioni di superficie saranno usati per un controllo di coerenza con la carta pedologica dell'area di Alberese curata dal Prof. Jan Sevink ${ }^{32}$. Tale procedura permetterà di identificare le porzioni di superficie stabili, asciutte e non interessate da successivi depositi alluvionali nelle varie fasi di frequentazione. In questo modo si potranno dare indicazioni preziose per le successive fasi di approfondimento del progetto.

\section{BIBLIOGRAFIA}

Arnoldus-Huyzendveld - Citter - Pizziolo 2016 = A. Arnoldus-Huyzendveld, C. Citter, G. Pizziolo, Predictivity-Postdictivity: a Theoretical Framework, in S. Campana, R. Scopigno, G. Carpentiero, M. Cirillo (ed.), CAA2015, Keep the revolution going, Proceedings of the 43rd Annual Conference on Computer Applications and Quantitative Methods in Archaeology, Oxford, 2016, p. 593-598.

Avanzini - Salvador 2014 = M. Avanzini, I. Salvador (a cura di), Antichi pastori-sopravvivenze, tradizione orale, storia, tracce nel paesaggio e archeologia, 2014.

Barfield 2002 = L. H. Barfield, L'Europa nel 3500 a.C.: una congiuntura tra diffusione e crisi ambientale?, in Il declino del mondo neolitico. Ricerche in Italia centro-settentrionale fra aspetti peninsulari, occidentali e nord-alpini, a cura di Ferrari, Visentini, Atti del Convegno (Pordenone 5-7 aprile 2001), Pordenone, 2002, p. 11-18. 
Barker 1977 = G. Barker, L'economia del bestiame a Luni, in A. Frova, Scavi di Luni II, Roma, 1977, p. 725-735.

Barker 1986 = G. Barker, L'archeologia del paesaggio italiano: nuovi orientamenti e recenti esperienze, in Archeologia Medievale, III, 1986, p. 7-29.

Barker 1995= G. Barker, The Biferno Valley: An Archaeological History of a Mediterranean Landscape the Archaeological and Geomorphological Record, Leicester, 1995

Barker - Maggi - Nisbet, 1990-1991 = G. Barker, R. Maggi, R. Nisbet, Archeologia della pastorizia nell'Europa meridionale, in Rivista di Studi Liguri, LVI-LVII, 1990-1991.

Barker - Hodges - Clark 1995= G. Barker, R. Hodges, G. Clark, A Mediterranean valley: landscape archaeology and Annales history in the Biferno Valley, Leicester, 1995.

Barsanti 1987 = D. Barsanti, Allevamento e transumanza in Toscana: pastori, bestiami e pascoli nei secoli XV-XIX, Firenze, 1987.

Bintliff 1999 = J. Bintliff, The Concepts of «Site» and «Off-site» Archaeology in Surface Artefact Survey, in M. Pasquinucci, F. Trément (éd.), Non-Destructive Techniques Applied to Landscape Archaeology, Oxford, 1999, p. 200-215.

Bintliff - Kuna - Venclova $2000=$ J. Bintliff, M. Kuna, N. Venclova, The future of artifact survey in Europe, Sheffield, 2000.

Bloch 1931 = M. Bloch, Les caractères originaux de l'histoire rurale française, Parigi, 1931.

Braudel 1949 = F. Braudel, La Méditerranée et le monde méditerranéen à l'époque de Philippe II, Parigi, 1949,2 vol.

Cambi et alii 2015 = F. Cambi, C. Citter, D. Cristoferi, M. De Silva, A. Guarducci, G. Macchi, G. Pizziolo, L. Sarti, E. Vanni, N. Volante, A. Zagli, A cross-disciplinary approach to the study of transhumance as territorial identity factor in a long-term perspective: the TraTTo project - southern Tuscany paths and pastures from Prehistory to the modern age, in Review of Historical Geography and Toponomastics, vol. X, 19-20, 2015, p. 85-98.

Cherubini 1974 = G. Cherubini, Signori, contadini, borghesi. Ricerche sulla società italiana del Basso Medioevo, Firenze, 1974.

Cherubini 1981 = G. Cherubini, Risorse, paesaggio ed utilizzazione agricola del territorio della Toscana sudoccidentale nei secc. XIV-XV, in Civiltà ed economia agricola in Toscana nei secc. XIV-XV: problemi della vita delle campagne del tardo medioevo, Pistoia, 1981.

Citter - Arnoldus-Huyzendveld 2007 = C. Citter, A. Arnoldus-Huyzendveld, Archeologia urbana a Grosseto. Origine e sviluppo di una citta medievale nella «Toscana delle citta:- deboli». Le ricerche 1997-2005, vol. I, La città di Grosseto nel contesto geografico della bassa valle dell'Ombrone; II, Edizione degli scavi urbani (1998-2005), Firenze, 2007.

Citter - Arnoldus-Huyzendveld 2011 = C. Citter, A. Arnoldus-Huyzendveld, Uso del suolo e sfruttamento delle risorse nella pianura grossetana nel Medioevo. Verso una storia del parcellario e del paesaggio agrario, Roma, 2011.

Citter - Arnoldus-Huyzendveld $2012=$ C. Citter, A. Arnoldus-Huyzendveld, New approaches to old issues: the application of predictive maps in archaeology. A case study: modeling the location of Grosseto's salt works from $700 B C$ to $A D$ 1200, in Medieval Settlement Research, 27, 2012, p. 1-11.

Cocchi Genik 1990 = D. Cocchi Genik, La pratica della transumanza dal Neolitico all'età del Bronzo nella Toscana settentrionale: evidenze archeologiche, in Rivista di Studi Liguri, LVI, 1990, p. 73-80. 
Collavini 1998 = S.M. Collavini, «Honorabilis domus et spetiosissimus comitatus»: gli Aldobrandeschi da conti a principi territoriali (secoli IX-XIII), Pisa, 1998.

Corbier 2006 = M. Corbier, La transhumance dans les pays de la Méditerranée antique, in P.-Y. Laffont (ed.), Transhumance et estivage en Occident des origines aux enjeux actuels, Tolosa, 2006, p. 67-82.

Cristoferi 2016 = D. Cristoferi, $I l$ «reame» di Siena: la costruzione della Dogana dei Paschi e lo sviluppo della transumanza in Maremma, Tesi di Dottorato, Università degli Studi di Siena, 2016.

De Grossi Mazzorin 1995 = J. De Grossi Mazzorin, Economie di allevamento in Italia centrale dalla media età del Bronzo alla fine dell'età del Ferro, in N. Christie (ed.), Settlement and Economy in Italy, 1500 BC-AD 1500. Papers of the Fifth Conference of Italian Archaeology, Exeter, 1995, p. 167-177.

De Grossi Mazzorin 2001 = J. De Grossi Mazzorin, Archaeozoology and Habitation Models: from a subsistence to a productive economy in Central Italy, in J.R., Brandt, L. Karlsonn, From huts to houses. Transformations of Ancient Societies. Proceedings of an International Seminar organized by the Norwegian and Swedish Institutes in Rome, 21-24 september 1997, Acta Instituti Romani Regni Sueciae Series 4, 56 (2001), p. 323-330.

De Grossi Mazzorin 2004 = J. De Grossi Mazzorin, Some considerations about the evolution of the animal exploitation in Central Italy from the Bronze Age to the Classical period, in B. Santillo Frizell (ed.), PECUS. Man and Animal in Antiquity. Proceedings of the conference at the Swedish institute in Rome, Roma, 2004, p. 38-49.

De Silva 2007 = M. De Silva, La cartografia storica per l'archeologia del paesaggio in ambiente GIS. Il caso dell'area grossetana, Tesi di Dottorato, Università degli Studi di Siena, Siena, 2007.

De Silva $2010=$ M. De Silva, Landscape of the Past: the Maremma Regional Park and the Grosseto Coastal Belt. Methodology and Technical Procedures, in F. Niccolucci, S. Herman (ed.), Beyond the ArtifactDigital Interpretation of the Past-Proceedings of CAA 2004, Prato 13-17 April 2004, Budapest, p. 166-170.

De Silva - Tarchi 2010 = M. De Silva, G. Tarchi, $i$ dati del Prospetto della Misura e della Stima del Catasto Leopoldino: prime considerazioni sugli assetti agrari e la rendita fondiaria nella Toscana dell'Ottocento, in G. Macchi Jánica (a cura di), Geografie del popolamento. Casi di studio, metodi e teorie, Siena, 2010, p. 321-330.

Di Fraia 2010 = T. Di Fraia, Aggiornamenti e riflessioni sul problema del sale nella preistoria e protostoria, in N. Negroni Catacchio (a cura di), L'alba dell'Etruria. Fenomeni di continuità e trasformazione nei secoli XIIVIII a.C. Ricerche e scavi, Atti del Nono Incontro di Studi di Preistoria e Protostoria in Etruria (Valentano [VT] - Pitigliano [GR], 12-14 settembre 2008), Milano, 2010, p. 597-607.

Foley 1981 = R.A. Foley, Off-site archaeology: an alternative approach for the short-sited, in I. Hodder, G. Isaac, N. Hammond (ed.), Pattern of the Past: Essays in Honour of David Clarke, Cambridge, 1981, p. 152-184.

Fugazzola - Pessina - Tinè 2005 = M.A. Fugazzola, A. Pessina, V. Tinè (a cura di), Il Neolitico in Italia: ricognizione, catalogazione e pubblicazione dei dati bibliografici, archivistici, materiali e monumentali, Firenze, 2005, 3 vol.

Gabba 1985 = E. Gabba, La transumanza nell'Italia romana: evidenze e problemi, qualche prospettiva per l'età altomedievale, in L'Uomo di fronte al mondo animale nell'alto Medioevo, 1985, p. 373-389.

Ginatempo 1988 = M. Ginatempo, Crisi di un territorio: il popolamento della Toscana senese alla fine del Medioevo, Firenze, 1988.

Imberciadori 2002 = I. Imberciadori, Il primo Statuto della Dogana dei Paschi maremmani, in I. Imberciadori, Studi su Amiata e maremma, Firenze, 2002, p. 97-125. 
Imberciadori 2002 = I. Imberciadori, Il reame della Repubblica senese, in I. Imberciadori, Studi su Amiata e maremma, Firenze, 2002, p. 71-79.

Marcaccini - Calzolai 2003 = P. Marcaccini, L. Calzolai, i percorsi della transumanza in Toscana, Firenze, 2003.

Mariotti Lippi et alii 2017 = M. Mariotti Lippi, L. Pisaneschi, L. Sarti, M. Lari, J. Moggi-Cecchi, Insights into the Copper-Bronze Age diet in Central Italy: Plant microremains in dental calculus from Grotta dello Scoglietto (Southern Tuscany, Italy), in Journal of Archaeological Science: Reports, 15 (2017), p. 30-39.

Marrara 1961 = D. Marrara, Storia istituzionale della Maremma senese. Principi e istituti di governo del territorio grossetano dall'età carolingia all'unificazione d'Italia, Siena, 1961.

Piccinni 1989 = G. Piccinni, L'Amiata nel contesto della montagna toscana: ambiente, produzione, società nel tardo Medioevo, in M. Ascheri, W. Kurze (a cura di), L'Amiata nel medioevo, Roma, 1989, p. 197-215.

Pinto 1982 = G. Pinto, La Toscana nel tardo medioevo. Ambiente, economia rurale, società, Firenze, 1982.

Pizziolo - Volante 2015 = G. Pizziolo, N. Volante, Landscape changes and site discovery potential: predictive criteria and field survey strategies for prehistoric contexts, in G. Pizziolo, L. Sarti, Predicting prehistory. Predictive models and field research methods for detecting prehistoric contexts, Firenze, 2015, p. 133-151.

Poesini 2012 = S. Poesini, La produzione ceramica di Punta degli Stretti (Orbetello, GR): aggiornamento degli studi, in N. Negroni Catacchio (a cura di), L'Etruria dal Paleolitico al Primo Ferro. Stato delle ricerche, Atti del decimo incontro di studi (Valentano [VT]-Pitigliano [GR], 10-12 Settembre 2010), Milano, 2012, p. 553-566.

Poggesi - Pessina 2015 = G. Poggesi, A. Pessina, Presentazioni, in A. Sebastiano, E. Chirico, M. Colombini, M. Cygielman (a cura di), Diana Umbronensis a Scoglietto. Santuario, Territorio e Cultura Materiale (200 a.C.-550 d.C.), Oxford, 2015, p. IV.

Sarti 2011 = L. Sarti, Grosseto. Alberese, Grotta dello Scoglietto: ricerche 2011, in Notiziario S.B.A.T., 7 (2011), p. 355-357.

Sarti 2014 = L. Sarti, Grotta dello Scoglietto (Alberese, Grosseto): aggiornamento sulle nuove ricerche, in Atti PPE, 11 (2014), p. 615-624.

Sebastiani - Chirico - Colombini - Cygielman 2015 = A. Sebastiani, E. Chirico, M. Colombini, M. Cygielman (a cura di), Diana Umbronensis a Scoglietto. Santuario, Territorio e Cultura Materiale (200 a.C. - 550 d.C.), Oxford, 2015.

Vaccaro 2007 = E. Vaccaro, L'occupazione tardoantica delle grotte dello Scoglietto e di Spaccasasso nei monti dell'Uccellina (GR), in C. Cavanna (a cura di), La Preistoria nelle grotte del parco naturale della maremma. Atti del museo di storia naturale della Maremma supplemento al n. 22, Grosseto, 2007, p. 227-242.

Vanni 2014 = E. Vanni, Sistemi agro-silvo-pastorali in un contesto dell'Etruria costiera. Aspetti conservativi del paesaggio in una prospettiva di lunga durata, Tesi di Dottorato, Università di Foggia, 2014.

Vanni - Cambi 2015 = E. Vanni, F. Cambi, Sale e transumanza. Approvvigionamento e mobilità in Etruria costiera tra Bronzo Finale e Medioevo, in F. Cambi, G. De Venuto, R. Goffedro (a cura di), $i$ pascoli, i campi, il mare. Paesaggi d'altura e di pianura in Italia dall'Età del Bronzo al Medioevo, Bari, 2015, p. 107-128. 
Volante $2003=$ N. Volante, La produzione vascolare dell'insediamento di Neto-Via Verga a Sesto Fiorentino tra la fine del Neolitico e la prima Età dei metalli, in Rivista di Scienze Preistoriche, LIII (2003), p. 375-504.

Volante - Pizziolo 2013 = N. Volante, G. Pizziolo, Grosseto. Alberese. Poggio di Spaccasasso, Sasso delle Donne. Fascia pedecollinare nord-occidentale dei Monti dell'Uccellina: campagne di scavo e survey 2012, in Notiziario della Soprintendenza dei Beni Archeologici della Toscana, 8, 2013, p. 550-552.

\section{NOTE}

1. Si veda per il Programme de recherche: www.efrome.it/la-recherche/programmes/detailprogramme/detail/la-transhumance-en-italie-centrale-de-la-protohistoire-a-nos-jours.html. Per il Progetto TraTTo: www.dssbc.unisi.it/it/ricerca/progetti-di-ricerca/progetto-tratto.

2. Si veda: Bloch 1931; Braudel 1949; Barker 1986; Barker 1995; Barker - Hodges - Clark 1995; Citter - Arnoldus-Huyzendveld 2011; Citter - Arnoldus-Huyzendveld 2012, ArnoldusHuyzendveld - Citter - Pizziolo 2016. Si veda a proposito dell'analisi regressiva per le pratiche transumanti Gabba 1985 e Corbier 2006.

3. Si veda tra gli altri: Marrara 1961; Cherubini 1981; Pinto 1982; Barsanti 1987; Ginatempo 1988; Piccinni 1989; Collavini 1998; Imberciadori 2002; Citter - Arnoldus-Huyzendveld 2007 e la relativa bibliografia.

4. Cristoferi 2016; Vanni 2014. Per la documentazione negli Archivi di Stato si veda la Sezione 2.

5. Si veda ad esempio il contributo delle indagini archeozoologiche proposto da De Grossi Mazzorin 1995, 2001, 2004.

6. Una serie di interrogativi, anche di carattere metodologico, e di proposte aperte furono formulate per i contesti italiani partendo da casi di studio alla Tavola Rotonda Internazionale Archeologia della pastorizia nell'Europa meridionale: atti della tavola rotonda internazionale (Maggi Nisbet - Barker 1990). Quella è stata la prima occasione di confronto e dibattito alla quale ancora possiamo fare riferimento per una identificazione dei primi marcatori e caratteristiche archeologiche riferibili alle prime forme di transumanza. In seno ai temi discussi Cocchi Genick, 1990 indicò per la Toscana settentrionale alcune specifiche sulle quali impostare la ricerca, fra queste ricordiamo: l'analisi distributiva su ambiti morfologici diversificati e l'individuazione di siti con frequenza ripetuta ma per brevi periodi.

7. Si veda ad esempio Fugazzola - Pessina - Tinè, 2005.

8. Ad esempio Poesini 2012. Per una riflessione più generale sul sale si veda Di Fraia 2010; Vanni Cambi 2015 cum bibl.

9. Barfield 2002.

10. Volante 2003.

11. Per una più ampia trattazione delle evidenze archeologiche dirette ed indirette collegabili alla transumanza prese in esame con il progetto TraTTo si veda Cambi et alii 2015.

12. Cristoferi 2016, p. 93-96.

13. Cristoferi 2016, p. 91-93, 492-506. Archivio di Stato di Firenze, Corporazioni religiose soppresse.

14. Cristoferi 2016, p. 88-92. Archivio di Stato di Siena, Statuti dello Stato; Capitoli.

15. Cristoferi 2016, p. 74-87. Archivio di Stato di Siena, Biccherna; Concistoro; Consiglio Generale; Gabella; Regolatori; Statuti di Siena; Dogana dei Paschi.

16. Si veda Archivio di Stato di Firenze: Mediceo del Principato, 2014; Mediceo del Principato, 2070; Consiglio di Reggenza, 325; Segreteria di Finanze. Affari prima del 1788, 717 e 723. Archivio di Stato di Siena, MS D 82-86; Quattro conservatori, 1702.

17. Cfr. ad esempio Cambi et alii 2015. 
18. A titolo esemplificativo si vedano gli atti della tavola rotonda Antichi pastori - sopravvivenze, tradizione orale, storia, tracce nel paesaggio e archeologia (Avanzini - Salvador2014) in cui i casi studio presentati sono per la maggior parte svolti utilizzando sistemi GIS in grado di confrontare fonti e dati di diversa tipologia.

19. Tutti i servizi WMS della Regione Toscana sono condivisi sulla base di licenze Creative Commons (CC). Servizio OGC di tipo WMS fornito da Regione Toscana (Sistema Informativo Territoriale ed Ambientale). i dati condivisi tramite GeoScopio_WMS sono in parte di proprietà della Regione Toscana, in parte sono prodotti da altri Enti pubblici, disponibili in copia o come elaborazione dei dati originali detenuti dai vari Enti proprietari. Scopo della loro pubblicazione è principalmente divulgativo e, se non diversamente specificato, non ha carattere di ufficialità.

20. Il Geoportale Nazionale è il punto di accesso nazionale disponibile tramite il sito del Ministero dell'Ambiente per gli scopi della direttiva INSPIRE consentendo quindi alle autorità competenti, parti terze e privati cittadini di ricercare le informazioni territoriali disponibili: http:// www.pcn.minambiente.it/.

21. Si veda a titolo esemplificativo De Silva 2007; De Silva 2010; De Silva - Tarchi 2010.

22. Il progetto CaStoRe, promosso dalla Regione Toscana è stato realizzato in collaborazione con gli Archivi di Stato toscani e ha riguardato la riproduzione digitale di oltre 12.000 mappe catastali ottocentesche, la loro schedatura analitica e la loro georeferenziazione (http:// web.rete.toscana.it/castoreapp/).

23. Marcaccini - Calzolai (2003).

24. Gli elaborati presenti nel volume utilizzano come base cartografica i fogli 1:100.000 della carta topografica IGMI. Su questo sfondo sono riportati i tracciati della viabilità legata alla transumanza oltre alle informazioni rappresentate con diverse simbologie relative a luoghi di sosta, dogane, valichi etc.

25. Soprintendenza Archeologia, Belle Arti e Paesaggio per le Province di Siena, Grosseto e Arezzo.

26. A titolo esemplificativo si veda Pizziolo - Volante 2015 cum bibl.; Volante - Pizziolo 2013, Citter - Arnoldus-Huyzeveld 2011 cum bibl.; Vaccaro et alii 2009.

27. Per un commento su questi aspetti si veda Poggesi - Pessina 2015.

28. Marcaccini - Calzolai 2003; Cristoferi 2016.

29. Recentemente sono stati ripresi gli scavi alla Grotta dello Scoglietto. Le indagini hanno evidenziato la continuità di frequentazione del sito dal Neolitico. Per alcune cavità le vicende paleo ambientali hanno probabilmente compromesso la conservazione di eventuali depositi archeologici specialmente delle fasi più antiche diversamente dalla Grotta dello Scoglietto che però non sembra restituire evidenze collegabili a pratiche pastorali (Sarti 2011; Sarti 2014; Mariotti et alii 2017; Vaccaro 2007).

30. È interessante notare che la discussione sul concetto di off site, a partire da quella proposta da Foley 1981 o dalle considerazioni emerse in Bintliff - Kuna - Venclova 2000 si è sviluppata anche in rapporto alle modalità e strategie di survey.

31. Si veda ad esempio Bintliff 1999.

32. Si ringrazia a questo proposito il Prof. Jan Senvink (Universiteit Amsterdam) che ci ha permesso la consultazione della cartografia pedologica di dettaglio redatta dal suo gruppo di lavoro e ha fornito preziose indicazioni e suggerimenti in occasione di visite durante le indagini sul campo. 


\section{INDICE}

institutions Dipartimento di Scienze Storiche e dei Beni Culturali dell'Università degli Studi di Siena, École française de Rome.

Mots-clés : ricognizione archeologica, percorsi storici, longue durée, Maremma toscana, transumanza

Keywords : archaeological survey, historical routes, long-run, Southern Tuscany, transhumance

\section{AUTORI}

\section{GIOVANNA PIZZIOLO}

Università degli Studi di Siena - pizziolo@unisi.it

MICHELE DE SILVA

Università degli Studi di Siena - mdesilva@unifi.it

\section{NICOLETTA VOLANTE}

Università degli Studi di Siena - nicoletta.volante@unisi.it

\section{DAVIDE CRISTOFERI}

Universiteit Gent, Università degli Studi di Siena - Davide.Cristoferi@UGent.be 